Proyecciones Journal of Mathematics

Vol. 37, No 4, pp. 637-681, December 2018.

Universidad Católica del Norte

Antofagasta - Chile

\title{
Fuzzy soft attribute correlation coefficient and application to data of human trafficking
}

\author{
Santanu Acharjee \\ Debraj Roy College, India \\ Diganta Jyoti Sarma \\ Central Institute of Technology, India \\ Robert A. Hanneman \\ University of California-Riverside, U.S.A. \\ John N. Mordeson \\ Creighton University, U.S.A. \\ and \\ Davender S. Malik \\ Creighton University, U.S.A. \\ Received: January 2018. Accepted : April 2018
}

Dedicated to Late Prof. Lotfi A. Zadeh

\begin{abstract}
In this paper, we introduce fuzzy soft attribute correlation coefficient and apply it to find the correlation between vulnerability and government response of various countries related to human trafficking based on six regions with the help of data from "The Global Slavery Index 2016". Comparison of fuzzy soft attribute correlation coefficients is done with the conventional analysis of sociology by calculating Pearson's zero-order product-moment correlations. Along with these, some fundamental concepts of mathematical statistics are developed with respect to fuzzy soft set.
\end{abstract}

2010 AMS Subject Classifications : 03E72, 03E99, 62A86, 62A99, 91099.

Keywords : Fuzzy soft set; correlation coefficient; $\alpha$-cut; soft set; vulnerability; human trafficking. 


\section{Introduction}

A mathematical theory is based on various abstract thoughts. One has full freedom to establish certain environments by neglecting many facts. For example in physics, we often neglect the frictional effect of air on a free falling body, but this fact is fully impossible in real life. Similarly, other branches like medical science, economics, engineering, social sciences, etc. are full of uncertainties. Molodtsov [12] introduced the concept of soft set theory in the year 1999 and investigated various applications in game theory, smoothness of functions, operation research, Perron integration, probability theory, the theory of measurement, etc.

Later Maji et al. [10] defined various operations on soft sets to study some of the fundamental properties. Pei and Miao [14], Chen [3] pointed out errors in some of the results of Maji et al. [10] and introduced some new notions and properties. At present, investigations of different properties and applications of soft set theory have attracted many researchers from various backgrounds. Since then many applications of soft set theory can be found in other branches of science and social science. Fuzzy soft set was introduced by Maji et al. [9] as a hybrid structure of soft set with fuzzy set. Fuzzy aspects of sociological researches can be found in $[8,6]$.

Trafficking in persons has been defined as the recruitment, transportation, transfer, harboring or receipt of persons by means of threat or use of force or other forms of coercion, of abduction, of fraud, of deception, of the abuse of power or of a position of vulnerability or the giving or receiving of payments or benefits to achieve the consent of a person having control over another person, for the purpose of exploitation [6]. It is one of the most heinous crimes of our times.

Accurate data concerning the flow of trafficking in persons is impossible to obtain due to the nature of the problem. The goal of the trafficker is to be undetected and to deceive. Besides the scale of the trafficking, there are many other reasons for the scarcity of data. Among these are the victims's reluctance to report crimes or testify for fear of reprisals, disincentives, both structural and legal, for law enforcement to act against traffickers, a lack of harmony among existing data sources, and an unwillingness of some countries and agencies to share data. Thus, concepts from the mathematics of uncertainty provide a valuable way to study the problems of human 
trafficking.

The data on vulnerability and government response that appears in our application is taken from [4]. "The Global Slavery Index 2016" is an annual study of worldwide slavery conditions by country published by the Walk Free Foundation. In 2016, the study estimated a total of 45.8 million people to be in some form of modern slavery in 167 countries. The report includes three data points for each country, national estimates of the prevalence of modern slavery, vulnerability measures, and assessment of the strength of government response. The index pioneered the use of random-sampled nationally-representative surveys to estimate prevalence. This included commissioning seven such surveys in 2014 and a further 19 surveys through Gallop World Poll in 2015.

The goal of this paper is to develop a method to compare the relationship of two fuzzy soft sets by using techniques from fuzzy soft set theory. We do this by developing a notion of fuzzy soft attribute correlation coefficient. This correlation coefficient is constructed by using the attributes of a fuzzy soft set and $\alpha$-cuts with respect to utility. We apply our results to determine the relationship between vulnerability and government response averages of countries making up a particular region. The regions are determined from combining certain regions defined in [15]. In [15], the size of the flow of human trafficking between countries is given linguistically. By using the regions in [15], this allows for the development of future researches involving the concepts from both [4] and [15]. One may refer to $[1,13]$ for the recent research related to the mathematics of uncertainties and human trafficking.

In Section 3, we discuss the construction of a fuzzy soft representation with respect to attributes and level cuts along with foundational notions of fuzzy soft statistics. In Section 4, we introduce fuzzy soft attribute correlation coefficients in terms of level sets. We show that The Americas and Europe have the highest correlation levels in this case. In Section 7, we give the fuzzy soft attribute correlation coefficient of the complement of vulnerability and government response in terms of level sets. The importance of the findings here is due to the fact that numbers in [4] representing the vulnerability of countries are high if the vulnerability is high and the numbers representing a country's government response are high if the response is high. One would be particularly interested in knowing the relationship 
640 S. Acharjee, D.J. Sarma, R.A. Hanneman, J.N. Mordeson and D.S. Malik

between low vulnerability ratings and high government response ratings. We show that The Americas and Europe have the highest correlation levels in this case also. In fact, the correlation levels for the complement of vulnerability and government response are higher than those for vulnerability and government response.

\section{Preliminaries}

Following definitions are due to Çăgman et al. [2].

Definition 2.1. [2] A soft set $F_{A}$ on the universe $U$ is defined by the set of ordered pairs $F_{A}=\left\{\left(x, f_{A}(x): x \in E, f_{A}(x) \in P(U)\right\}\right.$, where $f_{A}: E \rightarrow P(U)$ such that $f_{A}(x)=\emptyset$ if $x \notin A$.

Here, $f_{A}$ is called an approximate function of the soft set $F_{A}$. The value of $f_{A}(x)$ may be arbitrary. Some of them may be empty, some may have nonempty intersection. We will denote the set of all soft sets over $U$ as $S(U)$.

Definition 2.2. [2] Let $F_{A} \in S(U)$. If $f_{A}(x)=\emptyset$ for all $x \in E$, then $F_{A}$ is called a soft empty set, denoted by $F_{\emptyset}$.

$f_{A}(x)=\emptyset$ means there is no element in $U$ related to the parameter $x \in E$. Therefore, we do not display such elements in the soft sets, as it is meaningless to consider such parameters.

Definition 2.3. [2] Let $F_{A} \in S(U)$. If $f_{A}(x)=U$ for all $x \in A$, then $F_{A}$ is called an $A$-universal soft set, denoted by $F_{\widetilde{A}}$.

If $A=E$, then the $A$-universal soft set is denoted by $F_{\widetilde{E}}$.

Definition 2.4. [2] Let $F_{A}, F_{B} \in S(U)$. Then $F_{A}$ is a soft subset of $F_{B}$, denoted by $F_{A} \widetilde{\subseteq} F_{B}$, if $f_{A}(x) \subseteq f_{B}(x)$ for all $x \in E$.

Definition 2.5. [2] Let $F_{A}, F_{B} \in S(U)$. Then $F_{A}$ and $F_{B}$ are soft equal, denoted by $F_{A}=F_{B}$, if and only if $f_{A}(x)=f_{B}(x)$ for all $x \in E$.

Definition 2.6. [2] Let $F_{A}, F_{B} \in S(U)$. Then, the soft union $F_{A} \widetilde{\cup} F_{B}$, the 
soft intersection $F_{A} \widetilde{\cap} F_{B}$ and the soft difference $F_{A} \widetilde{\backslash} F_{B}$ of $F_{A}$ and $F_{B}$ are defined by the approximation functions

$$
f_{A \widetilde{\cup} B}(x)=f_{A}(x) \cup f_{B}(x), f_{A \widetilde{\cap} B}(x)=f_{A}(x) \cap f_{B}(x) \text { and } f_{A \backslash B}(x)=
$$
$f_{A}(x) \backslash f_{B}(x)$ respectively and the soft complement $F_{A}^{\widetilde{c}}$ of $F_{A}$ is defined by the approximate function, $f_{A}^{\widetilde{c}}(x)=f_{A}^{c}(x)$, where $f_{A}^{c}(x)$ is the compliment of the set $f_{A}(x)$; that is $f_{A}^{c}(x)=U \backslash f_{A}(x)$ for all $x \in E$.

It is easy to see that $\left(F_{A}^{\widetilde{c}}\right)^{\widetilde{c}}=F_{A}$ and $\widetilde{F_{\emptyset}}=F_{\widetilde{E}}$.

Example 2.1. [2] Let us consider a universe $U=\{a, b, c\}$ and $E=$ $\left\{e_{1}, e_{2}, e_{3}, e_{4}\right\}$. Let $A=\left\{e_{1}, e_{2}, e_{3}\right\}$. We define a soft set $(F, A)=\left\{\left(e_{1},\{a, b\}\right),\left(e_{2},\{a, c\}\right),\left(e_{3},\{a, b, c\}\right)\right\}$.

Then, the representation of $(F, A)$ in tabular form is shown in Table 1:

\begin{tabular}{|c|c|c|c|}
\hline & $F\left(e_{1}\right)$ & $F\left(e_{2}\right)$ & $F\left(e_{3}\right)$ \\
\hline$a$ & 1 & 1 & 1 \\
$b$ & 1 & 0 & 1 \\
$c$ & 0 & 1 & 1 \\
\hline
\end{tabular}

Table 1

Definition 2.7. [9] Let $U$ be a universe and let $A$ be a set of parameters. The pair $(F, A)$ is called a fuzzy soft set over $U$, where $F: A \rightarrow[0,1]^{U}$.

$[0,1]^{U}$ denotes the set of all fuzzy sets of $U$.

Example 2.2. [11] Suppose that a fuzzy soft set $(F, E)$ describes attractiveness of the shirts which the authors are going to wear. $U=$ the set of all shirts under consideration $=\left\{x_{1}, x_{2}, x_{3}, x_{4}, x_{5}\right\}$. Let $I^{U}$ be the collection of all fuzzy subsets of $U$. Also, let $E=\{$ colourful,bright, cheap,warm $\}=$ $\left\{e_{1}, e_{2}, e_{3}, e_{4}\right\}$.

Let $F\left(e_{1}\right)=\left\{\frac{x_{1}}{0.5}, \frac{x_{2}}{0.9}, \frac{x_{3}}{0}, \frac{x_{4}}{0}, \frac{x_{5}}{0}\right\} ; F\left(e_{2}\right)=\left\{\frac{x_{1}}{1.0}, \frac{x_{2}}{0.8}, \frac{x_{3}}{0.7}, \frac{x_{4}}{0}, \frac{x_{5}}{0}\right\} ; F\left(e_{3}\right)=$ $\left\{\frac{x_{1}}{0}, \frac{x_{2}}{0}, \frac{x_{3}}{0}, \frac{x_{4}}{0.6}, \frac{x_{5}}{0}\right\} ; F\left(e_{4}\right)=\left\{\frac{x_{1}}{0}, \frac{x_{2}}{1}, \frac{x_{3}}{0}, \frac{x_{4}}{0}, \frac{x_{5}}{0.3}\right\}$

So, the fuzzy soft set $(F, E)$ is a family $\left\{F\left(e_{i}\right): i=1,2,3,4\right\}$ of $I^{U}$. 
642 S. Acharjee, D.J. Sarma, R.A. Hanneman, J.N. Mordeson and D.S. Malik

Definition 2.8. [11] For two fuzzy soft sets $(F, A)$ and $(G, B)$ over a common universe $U$, we say that $(F, A)$ is a fuzzy soft subset of $(G, B)$; denoted by $(F, A) \widetilde{\subseteq}(G, B)$ if (i) $A \subseteq B$ (ii) $F(e) \subseteq G(e)$.

Definition 2.9. [11] Union of two fuzzy soft sets $(F, A)$ and $(G, B)$ over a common universe $U$ is the fuzzy soft set $(H, C)$ denoted by $(F, A) \widetilde{\cup}(G, B)=$ $(H, C)$; where

$$
H(e)=F(e) \text { if } e \in A \backslash B ; G(e) \text { if } e \in B \backslash A ; F(e) \cup G(e) \text { if } e \in A \cap B \text {. }
$$

Definition 2.10. [11] For two fuzzy soft sets $(F, A)$ and $(G, B)$ over a common universe $U$ are said to be fuzzy soft equal if $(F, A)$ is a fuzzy soft subset of $(G, B)$ and $(G, B)$ is a fuzzy soft subset of $(F, A)$.

Definition 2.11. [11] A fuzzy soft set $(F, A)$ over $U$ is said to be null fuzzy soft set denoted by $\phi$, if $\forall e \in A ; F(e)=$ null fuzzy set of $U$.

Definition 2.12. [11] A fuzzy soft set $(F, A)$ over $U$ is said to be absolute fuzzy soft set denoted by $\widetilde{A}$, if $\forall e \in A, F(e)=U$.

Definition 2.13. [17] Restricted intersection of two fuzzy soft sets $(F, A)$ and $(G, B)$ over a common universe $U$ is the fuzzy soft set $(H, C)$ denoted by $(F, A) \widetilde{\cap}(G, B)=(H, C)$; where $C=A \cap B \neq \emptyset$ and $\forall e \in C ; H(e)=$ $F(e) \cap G(e)$.

Proposition 2.1. [16] Every fuzzy set can be considered as soft set.

Following definition is due to Zadeh [17] whenever $A, B$ are two fuzzy sets.

Definition 2.14. [17] $A$ is contained in $B$ (or, equivalently, $A$ is a subset of $B$, or $A$ is smaller than or equal to $B$ ) if and only if $f_{A} \leq f_{B}$. In symbols $A \subseteq B \Leftrightarrow f_{A} \leq f_{B}$. 


\section{Some fundamental statistical notions of fuzzy soft set with respect to attributes, utility and $\alpha$-cut}

In this section, we introduce fuzzy soft mean, $\alpha$-cut fuzzy soft standard deviation, etc. with examples and proved some theorems. Throughout this paper, we shall denote $I=\{1,2,3, \ldots, n\}$ and $\Delta$ is an index set.

\subsection{Fuzzy soft mean, $\alpha$-cut fuzzy soft standard deviation, etc.}

Definition 3.1. If $(F, A)$ be a fuzzy soft set over a universe $U$; where $F\left(e_{i}\right)$ is a fuzzy set for the attribute $e_{i} \in A$, then fuzzy soft mean of $(F, A)$ is denoted by $\widetilde{F_{A}}=\{(A, F(A))\}$ and $F(A)$ is defined as follows:

$F(A)=\left\{\frac{x_{1}}{\min \left\{\alpha_{1}^{i}\right\}}, \frac{x_{2}}{\min \left\{\alpha_{2}^{i}\right\}}, \frac{x_{3}}{\min \left\{\alpha_{3}^{i}\right\}}, \ldots, \frac{x_{k}}{\min \left\{\alpha_{k}^{i}\right\}}, \ldots\right\}$. Here, $\alpha_{k}^{i}$ is the membership value of $x_{k}$ associated with attributes $e_{i}$, where $k \in \Delta$ and $i \in I$.

Here, we consider minimum in $F(A)$ as we want to find the minimum of degrees of membership of $x_{k} \forall i \in I, k \in \Delta$. It is due to the fact that $\min \left\{\alpha_{k}^{i}\right\} \leq \alpha_{k}^{i} \forall e_{i} \in A$. Since, $\forall e_{i} \in A, F(A) \subseteq F\left(e_{i}\right)$ in the sense of fuzzy set, thus we have used the notion $\widetilde{F_{A}}=\{(A, F(A))\}$, rather than writing as $\widetilde{F_{A}}=\left\{\left(e_{i}, F(A)\right) \mid e_{i} \in A\right\}$.

If $0 \leq \alpha \leq 1$, then $\alpha \overrightarrow{F_{A}}$ is defined as $\alpha \overrightarrow{F_{A}}=\left(\alpha_{1}{ }^{\prime}, \alpha_{2}{ }^{\prime}, \alpha_{3}{ }^{\prime}, \ldots, \alpha_{n}{ }^{\prime}, \ldots\right)$, where $\alpha_{k}{ }^{\prime}=1$ if $\min \left\{\alpha_{k}{ }^{i}\right\} \geq \alpha$, otherwise 0 for $i \in I, k \in \Delta$

Example 3.1. Let us consider a fuzzy soft set $(F, A)=\left\{\left(e_{1},\left\{\frac{x_{1}}{0.9}, \frac{x_{2}}{0.8}, \frac{x_{3}}{0.3}\right\}\right)\right.$, $\left.\left(e_{2},\left\{\frac{x_{1}}{0.4}, \frac{x_{2}}{0.6}, \frac{x_{3}}{0.4}\right\}\right),\left(e_{3},\left\{\frac{x_{1}}{0.3}, \frac{x_{2}}{0.6}, \frac{x_{3}}{0.2}\right\}\right)\right\}$. Then, $\widetilde{F}_{A}=\left\{\left(A,\left\{\frac{x_{1}}{0.3}, \frac{x_{2}}{0.6}, \frac{x_{3}}{0.2}\right\}\right)\right\}$, where $A=\left\{e_{1}, e_{2}, e_{3}\right\}$ and $U=\left\{x_{1}, x_{2}, x_{3}\right\}$.

Thus, $0.3 \overrightarrow{F_{A}}=(1,1,0), 0.4 \overrightarrow{F_{A}}=(0,1,0), 0.5 \overrightarrow{F_{A}}=(0,1,0), 0.6 \overrightarrow{F_{A}}=$ $(0,1,0), 0.7 \overrightarrow{F_{A}}=(0,0,0)$ and so on.

Definition 3.2. Let us consider a fuzzy soft set $(F, A)$ where $e_{i} \in A$. Then, $\alpha \overrightarrow{F\left(e_{i}\right)}=\left(\beta_{1}{ }^{\prime}, \beta_{2}{ }^{\prime}, \beta_{3}{ }^{\prime}, \ldots, \beta_{n}{ }^{\prime}, \ldots\right)$ where $\beta_{j}{ }^{\prime}=1$ if $F_{j}\left(e_{i}\right)\left(x_{j}\right) \geq \alpha$, otherwise 0 . Here, $F_{j}\left(e_{i}\right)\left(x_{j}\right)$ denotes the membership value of $x_{j}$ in the $j^{\text {th }}$ place of $F\left(e_{i}\right)$. 
Example 3.2. Let us consider Example 3.1, where $0.3 \overrightarrow{F\left(e_{1}\right)}=(1,1,1)$, $0.6 \overrightarrow{F\left(e_{1}\right)}=(1,1,0), 0.6 \overrightarrow{F\left(e_{2}\right)}=(0,1,0)$ and so on.

Definition 3.3. Let $(F, A)$ be a fuzzy soft set then scale of $(F, A)$ is defined as $h=\max \left\{F\left(e_{i}\right)\left(x_{j}\right): e_{i} \in A, x_{j} \in U, i \in I, j \in \Delta\right\}$.

Definition 3.4. Let us consider a universe $U$ and a set of attributes $E$, where $A \subseteq E$ with $|A|=n$. If $(F, A)$ be a fuzzy soft set over $U$, then $\alpha$-cut fuzzy soft standard deviation of $(F, A)$ is denoted by $\sigma(\alpha \overrightarrow{(F, A)})$ and it is defined by $\sqrt{\frac{1}{n} \sum_{i \in I}\left\|\alpha \overrightarrow{F\left(e_{i}\right)}-\alpha \overrightarrow{F_{A}}\right\|^{2}}$, where $\left\|\alpha \overrightarrow{F\left(e_{i}\right)}-\alpha \overrightarrow{F_{A}}\right\|^{2}=$ $\left\langle\alpha \overrightarrow{F\left(e_{i}\right)}-\alpha \overrightarrow{F_{A}}, \alpha \overrightarrow{F\left(e_{i}\right)}-\alpha \overrightarrow{F_{A}}\right\rangle$.

Example 3.3. Let $(F, A)=\left\{\left(e_{1},\left\{\frac{x_{1}}{0.9}, \frac{x_{2}}{0.8}, \frac{x_{3}}{0.5}\right\}\right),\left(e_{2},\left\{\frac{x_{1}}{0.4}, \frac{x_{2}}{0.6}, \frac{x_{3}}{0.6}\right\}\right)\right.$, $\left.\left(e_{3},\left\{\frac{x_{1}}{0.3}, \frac{x_{2}}{0.6}, \frac{x_{3}}{0.2}\right\}\right)\right\}$. Then, $\widetilde{F_{A}}=\left\{\left(A,\left\{\frac{x_{1}}{0.3}, \frac{x_{2}}{0.6}, \frac{x_{3}}{0.2}\right\}\right)\right\}$, where $A=\left\{e_{1}, e_{2}, e_{3}\right\}$ and $U=\left\{x_{1}, x_{2}, x_{3}\right\}$.

Now, $0.3 \overrightarrow{F_{A}}=(1,1,0), 0.3 \overrightarrow{F\left(e_{1}\right)}=(1,1,1), 0.3 \overrightarrow{F\left(e_{2}\right)}=(1,1,1)$, and $0.3 \overrightarrow{F\left(e_{3}\right)}=(1,1,0)$. Then, $\sigma(0.3 \overrightarrow{(F, A)})=\sqrt{\frac{2}{3}}$.

\subsection{Concept of utility wise representation of fuzzy soft set}

Consider a fuzzy soft set $(F, A)$ over a universe $U$ and $\mathbf{R}$ is the set of real numbers. We define an $\alpha$-cut level utility function $\mu_{\alpha}: U \rightarrow \mathbf{R}$ as $x \succeq y \Longleftrightarrow \mu_{\alpha}(x) \geq \mu_{\alpha}(y)$ for $x, y \in U$ and so on with fundamental notions of utility representations of utility theory. Here, the notion $x \succeq y$ indicates that $x$ is strictly preferred and indifferent to $y$. Readers can find these notions in literature of utility theory and microeconomics.

Without any presumption of fuzzy $\alpha$-cut, it must be mentioned that here we indicate $\alpha$-cut, which is based on utility. Thus, it is easy to find that our $\alpha$-cut doesn't generate a set with elements from $x_{k}$ 's as usually we do in fuzzy $\alpha$-cut. Readers should not be confused with this notion.

If $F_{j}\left(e_{i}\right)\left(x_{j}\right) \geq \alpha$ for $x_{j} \in U$ and $e_{i} \in A$, then utility wise representation of $x_{j}$ in $F\left(e_{i}\right)$ is shown in Table 2. 


\begin{tabular}{|c|c|c|}
\hline & $F\left(e_{i}\right)$ & $F\left(e_{i}\right)^{c}$ \\
\hline$x_{j}^{\alpha}$ & $\mu\left(x_{j}\right)$ & $1-\mu\left(x_{j}\right)$ \\
\hline
\end{tabular}

Table 2

If $F_{j}\left(e_{i}\right)\left(x_{j}\right)<\alpha$, then we assume $\mu\left(x_{j}\right)=0$ for the particular case beyond any presumption of $\mu\left(x_{j}\right)$. Here, $x_{j}^{\alpha}$ denotes $x_{j} \in U$ with $\alpha$-cut level utility.

Example 3.4. Let us consider Example 3.3. We define a 0.3-cut level utility function $\mu_{0.3}: U \rightarrow \mathbf{R}$ as $\mu_{0.3}\left(x_{1}\right)=5, \mu_{0.3}\left(x_{2}\right)=-2$ and $\mu_{0.3}\left(x_{3}\right)=3$. Thus, utility wise representation of $(F, A)$ at 0.3 -cut level utility is shown in Table 3.

\begin{tabular}{|c|c|c|c|}
\hline & $F\left(e_{1}\right)$ & $F\left(e_{2}\right)$ & $F\left(e_{2}\right)$ \\
\hline$x_{1}^{0.3}$ & 5 & 5 & 5 \\
$x_{2}^{0.3}$ & -2 & -2 & -2 \\
$x_{3}^{0.3}$ & 3 & 3 & 0 \\
\hline
\end{tabular}

Table 3

Then, we call $(5,-2,3)$ as the origin of $(F, A)$ at 0.3 -cut level utility and this concept can be extended for any fuzzy soft set.

It is important to note that the origin of a fuzzy soft set is not fixed as the concept of classical mathematics or statistics. It is based on one's choice and $\alpha$-cut level of utility.

\subsection{Generating process of a new fuzzy soft set from the old one with respect to $\alpha$-cut and utility}

(i) $\alpha$-cut generated fuzzy soft set

Consider a fuzzy soft set $(F, A)$ whose $\alpha$-cut level representation is denoted by $\alpha \overrightarrow{(F, A)}$ over a universe $U$ with elements $x_{i}, i \in \Delta$ and $e_{j} \in A, j \in$ $I$.

Then, we can generate a new fuzzy soft set $\left(G_{F}, A\right)_{\alpha}$ with $\alpha$-cut level of representation $\alpha \overrightarrow{\left(G_{F}, A\right)}$ if $\left(\alpha_{1}, \alpha_{2}, \ldots, \alpha_{i}, \ldots\right)+\alpha \overrightarrow{(F, A)}$ exists, where 
646 S. Acharjee, D.J. Sarma, R.A. Hanneman, J.N. Mordeson and D.S. Malik

$\left(\alpha_{1}, \alpha_{2}, \ldots, \alpha_{i}, \ldots\right) \neq(0,0,0, \ldots, 0, \ldots), \alpha_{i} \in \mathbf{R}$ and $i \in \Delta$

Now, we discuss the generating process. We define an $\alpha$-cut level utility function $\mu_{\alpha}: U \rightarrow \mathbf{R}$ such that if $\alpha_{i}+F_{i}\left(e_{j}\right) \geq \mu_{\alpha}\left(x_{i}\right), i \in \Delta, j \in I$; then $x_{i} \in G_{i}\left(e_{j}\right)$ with the membership value minimum of $\{$ membership value of $x_{i}$ in $\left.F\left(e_{j}\right), \alpha\right\}$, otherwise, $x_{i} \notin G_{i}\left(e_{j}\right)$.

Here, $\left(\alpha_{1}, \alpha_{2}, \ldots, \alpha_{i}, \ldots\right)+\alpha \overrightarrow{(F, A)}=\left\{\left(\alpha_{1}, \alpha_{2}, \ldots, \alpha_{i}, \ldots\right)\right.$
$\left.+\alpha \overrightarrow{F\left(e_{1}\right)},\left(\alpha_{1}, \alpha_{2}, \ldots, \alpha_{i}, ..\right)+\alpha \overrightarrow{F\left(e_{2}\right)}, \ldots,\left(\alpha_{1}, \alpha_{2}, \ldots, \alpha_{i}, \ldots\right)+\alpha \overrightarrow{F\left(e_{n}\right)}\right\}$, where
$i \in \Delta, j \in I$.

The new fuzzy soft set $\left(G_{F}, A\right)_{\alpha}$ with representation $\alpha \overrightarrow{\left(G_{F}, A\right)}$ $=\left(\alpha_{1}, \alpha_{2}, \ldots, \alpha_{i}, \ldots\right)+\alpha \overrightarrow{(F, A)}$ is called an $\alpha$-cut generated fuzzy soft set of $(F, A)$. The fuzzy soft mean of $\left(G_{F}, A\right)_{\alpha}$ is denoted by $\widetilde{G_{A}}$ with $\alpha$-cut level of representation $\alpha \overrightarrow{G_{A}}$.

(ii) $\alpha$-cut generated fuzzy soft set with scale

Let the scale of a fuzzy soft set $(F, A)$ be $h$. Then, similarly as discussed above we can generate a new $\alpha$-cut generated fuzzy soft set $\left(G_{F}, A\right)_{\alpha}^{h}$ with representation $\alpha \overrightarrow{\left(G_{F}, A\right)_{h}}=\frac{\alpha \overrightarrow{\left(G_{F}, A\right)}}{h}$.

The fuzzy soft mean of $\left(G_{F}, A\right)_{\alpha}^{h}$ is denoted by $\widetilde{G_{A}{ }^{h}}$ with $\alpha$-cut level representation of fuzzy soft mean of $\left(G_{F}, A\right)_{\alpha}^{h}$ as $\alpha \overrightarrow{G_{A}^{h}}$.

Example 3.5. Let us consider a fuzzy soft set $(F, A)$ $=\left\{\left(e_{1},\left\{\frac{x_{1}}{0.4}, \frac{x_{2}}{0.8}, \frac{x_{3}}{0.5}\right\}\right),\left(e_{2},\left\{\frac{x_{1}}{0.6}, \frac{x_{2}}{0.2}, \frac{x_{3}}{0.2}\right\}\right)\right\}$. We define a 0.3 -cut level of utility function $\mu_{0.3}: U \rightarrow \mathbf{R}$ as $\mu_{0.3}\left(x_{1}\right)=5, \mu_{0.3}\left(x_{2}\right)=-2$ and $\mu_{0.3}\left(x_{3}\right)=3$.

Let $\left(\alpha_{1}, \alpha_{2}, \alpha_{3}\right)=(1,4,5)$, then $(1,4,5)+0.3 \overrightarrow{(F, A)}$ $=\{(1,4,5)+(1,1,1),(1,4,5)+(1,0,0)\}=\{(2,5,6),(2,4,5)\}$.

Then, we have $\left(G_{F}, A\right)_{0.3}=\left\{\left(e_{1},\left\{\frac{x_{1}}{0}, \frac{x_{2}}{0.3}, \frac{x_{3}}{0.3}\right\}\right),\left(e_{2},\left\{\frac{x_{1}}{0}, \frac{x_{2}}{0.2}, \frac{x_{3}}{0.2}\right\}\right)\right\}$.

Theorem 3.1. Let $(F, A)$ be a fuzzy soft set over $U$ and $\left(G_{F}, A\right)_{\alpha}$ be an $\alpha$-cut generated fuzzy soft set of $(F, A)$, then $\sigma(\alpha \overrightarrow{(F, A)})=\sigma\left(\alpha \overrightarrow{\left(G_{F}, A\right)}\right)$ 
Proof: Let $\left(\alpha_{1}, \alpha_{2}, \alpha_{3}, \ldots, \alpha_{i}, \ldots\right) \neq(0,0,0, \ldots, 0, \ldots)$, where $\alpha_{i} \in \mathbf{R}, i \in \Delta$.

We define an $\alpha$-cut level utility function $\mu_{\alpha}: U \rightarrow \mathbf{R}$ as $\mu_{\alpha}\left(x_{i}\right)=\beta_{i}$ such that $x_{i} \in G_{i}\left(e_{j}\right)$ if $\alpha_{i}+F_{i}\left(e_{j}\right) \geq \beta_{i}$, where $i \in \Delta, j \in I$. Here, $x_{i} \in G_{i}\left(e_{j}\right)$ indicates that $x_{i}$ is in $i^{\text {th }}$ place of $G\left(e_{j}\right)$ with membership value.

Then, $\alpha \overrightarrow{G_{A}}=\left(\min \left\{\alpha_{1}+\alpha F_{1}\left(e_{1}\right), \alpha_{1}+\alpha F_{1}\left(e_{2}\right), \ldots, \alpha_{1}+\alpha F_{1}\left(e_{n}\right)\right\}, \min \left\{\alpha_{2}+\right.\right.$ $\left.\alpha F_{2}\left(e_{1}\right), \alpha_{2}+\alpha F_{2}\left(e_{2}\right), \ldots, \alpha_{2}+\alpha F_{2}\left(e_{n}\right)\right\}, \ldots, \min \left\{\alpha_{i}+\alpha F_{i}\left(e_{1}\right), \alpha_{i}+\alpha F_{i}\left(e_{2}\right), \ldots\right.$, $\left.\left.\alpha_{i}+\alpha F_{i}\left(e_{n}\right)\right\}, \ldots\right)$, where $\alpha F_{i}\left(e_{n}\right)$ indicates the $i^{t h}$-coordinate of $\alpha \overrightarrow{F\left(e_{n}\right)}$ with respect to $x_{i}$.

$$
=\left(\alpha_{1}+\alpha F_{1}\left(e_{j_{1}}\right), \alpha_{2}+\alpha F_{2}\left(e_{j_{2}}\right), \alpha_{3}+\alpha F_{3}\left(e_{j_{3}}\right), \ldots, \alpha_{i}+\alpha F_{i}\left(e_{j_{i}}\right), \ldots\right) \text { (say), }
$$
where each $j_{i} \in I$.

Now, $\alpha \overrightarrow{G\left(e_{j}\right)}-\alpha \overrightarrow{G_{A}}=\left(\alpha_{1}+\alpha F_{1}\left(e_{j}\right), \alpha_{2}+\alpha F_{2}\left(e_{j}\right), \ldots, \alpha_{i}+\alpha F_{i}\left(e_{j}\right), \ldots\right)-\left(\alpha_{1}+\right.$ $\left.\alpha F_{1}\left(e_{j_{1}}\right), \alpha_{2}+\alpha F_{2}\left(e_{j_{2}}\right), \alpha_{3}+\alpha F_{3}\left(e_{j_{3}}\right), \ldots, \alpha_{i}+\alpha F_{i}\left(e_{j_{i}}\right), \ldots\right)$.

$$
\begin{aligned}
& =\left(\alpha F_{1}\left(e_{j}\right)-\alpha F_{1}\left(e_{j_{1}}\right), \alpha F_{2}\left(e_{j}\right)-\alpha F_{2}\left(e_{j_{2}}\right), \ldots, \alpha F_{i}\left(e_{j}\right)-\alpha F_{i}\left(e_{j_{i}}\right), \ldots\right) \\
& =\alpha \overrightarrow{F\left(e_{j}\right)}-\alpha \overrightarrow{F_{A}}
\end{aligned}
$$

Now, $\left\|\alpha \overrightarrow{G\left(e_{j}\right)}-\alpha \overrightarrow{G_{A}}\right\|^{2}=\left\langle\alpha \overrightarrow{G\left(e_{j}\right)}-\alpha \overrightarrow{G_{A}}, \alpha \overrightarrow{G\left(e_{j}\right)}-\alpha \overrightarrow{G_{A}}\right\rangle$.

$$
\begin{aligned}
& =\left\langle\alpha \overrightarrow{F\left(e_{j}\right)}-\alpha \overrightarrow{F_{A}}, \alpha \overrightarrow{F\left(e_{j}\right)}-\alpha \overrightarrow{F_{A}}\right\rangle \\
& =\left\|\alpha \overrightarrow{F\left(e_{j}\right)}-\alpha \overrightarrow{F_{A}}\right\|^{2}
\end{aligned}
$$

Thus, $\sigma(\alpha \overrightarrow{(F, A)})=\sigma\left(\alpha \overrightarrow{\left(G_{F}, A\right)}\right)$

Corollary 3.1. Let $(F, A)$ be a fuzzy soft set over $U$ and $\left(G_{F}, A\right)_{\alpha}$, $\left(H_{F}, A\right)_{\alpha}$ are two distinct $\alpha$-cut generated fuzzy soft sets of $(F, A)$, then $\sigma(\alpha \overrightarrow{(F, A)})=\sigma\left(\alpha \overrightarrow{\left(G_{F}, A\right)}\right)=\sigma\left(\alpha \overrightarrow{\left(H_{F}, A\right)}\right)$.

Thus, it can be said that $\alpha$-cut fuzzy soft standard deviation of an $\alpha$-cut generated fuzzy soft of any fuzzy soft set is independent of the choice of $\left(\alpha_{1}, \alpha_{2}, \ldots, \alpha_{i}, \ldots\right)$ and utility. 
648 S. Acharjee, D.J. Sarma, R.A. Hanneman, J.N. Mordeson and D.S. Malik

Example 3.6. Let us consider Example 3.5.

Here, $0.3 \overrightarrow{F_{A}}=(1,0,0), 0.3 \overrightarrow{F\left(e_{1}\right)}=(1,1,1), 0.3 \overrightarrow{F\left(e_{2}\right)}=(1,0,0)$, then $\sigma(0.3 \overrightarrow{(F, A)})=1$.

Again, $0.3 \overrightarrow{G_{A}}=(0,0,0), 0.3 \overrightarrow{G\left(e_{1}\right)}=(0,1,1), 0.3 \overrightarrow{F\left(e_{2}\right)}=(0,0,0)$, then $\sigma\left(0.3 \overrightarrow{\left(G_{F}, A\right)}\right)=1$.

The proof of the following theorem can be obtained by following the steps of Theorem 3.1 and using the concept of section " $\alpha$-cut generated fuzzy soft set with scale".

Theorem 3.2. Let $(F, A)$ be a fuzzy soft set over $U$ and $\left(G_{F}, A\right)_{\alpha}^{h}$ be an $\alpha$-cut generated fuzzy soft set of $(F, A)$, then $\sigma\left(\alpha{\overrightarrow{\left(G_{F}, A\right)}}_{h}\right)=\frac{1}{h} \sigma(\alpha \overrightarrow{(F, A)})$.

\subsection{Fuzzy soft coefficient of variation}

Definition 3.5. Let us consider a fuzzy soft set $(F, A)$ whose $\alpha$-cut level representation is denoted by $\alpha \overrightarrow{(F, A)}$ over a universe $U$. Then, $\alpha$-cut level fuzzy soft coefficient of variation is denoted by $\alpha F S C V \overrightarrow{(F, A)}$ and it is defined as $\alpha F S C V \overrightarrow{(F, A)}=\left\{\frac{\sigma(\alpha \overrightarrow{(F, A)})}{\left\|\alpha \overrightarrow{F_{A}}\right\|^{2}}\right\} \times 100$.

Theorem 3.3. Let $(F, A)$ be a fuzzy soft set over $U$, then $\alpha F S C V \overrightarrow{\left(G_{F}, A\right)}$ $=\left\{\frac{\sigma(\alpha \overrightarrow{(F, A)})}{\theta}\right\} \times 100$, where $\theta=\|\beta\|^{2}+2\left\langle\beta, \alpha \overrightarrow{F_{A}}\right\rangle+\left\|\alpha \overrightarrow{F_{A}}\right\|^{2}$ and $\beta=$ $\left(\alpha_{1}, \alpha_{2}, \alpha_{3}, \ldots, \alpha_{i}, \ldots\right)$.

Proof. From the definition, we know that $\alpha \overrightarrow{\left(G_{F}, A\right)}=\beta+\alpha \overrightarrow{(F, A)}$, where $\beta=\left(\alpha_{1}, \alpha_{2}, \alpha_{3}, \ldots, \alpha_{i}, \ldots\right)$. Thus, it is easy to find that $\alpha \overrightarrow{G_{A}}=\beta+\alpha \overrightarrow{F_{A}}$.

Thus, $\left\|\alpha \overrightarrow{G_{A}}\right\|^{2}=\|\beta\|^{2}+2\left\langle\beta, \alpha \overrightarrow{F_{A}}\right\rangle+\left\|\alpha \overrightarrow{F_{A}}\right\|^{2}$ (= = , say); since in our case $\left\langle\beta, \alpha \overrightarrow{F_{A}}\right\rangle=\left\langle\alpha \overrightarrow{F_{A}}, \beta\right\rangle$.

Again from Theorem 3.1., we have $\sigma(\alpha \overrightarrow{(F, A)})=\sigma\left(\alpha \overrightarrow{\left(G_{F}, A\right)}\right)$. Hence, Definition 3.5. yields the result. 
Theorem 3.4. Let $(F, A)$ be a fuzzy soft set over $U$, then $\alpha F S C V \overrightarrow{\left(G_{F}, A\right)_{h}}$ $=h \times \alpha F S C V \overrightarrow{\left(G_{F}, A\right)}$.

Example 3.7. Let us consider a fuzzy soft set $(F, A)=\left\{\left(e_{1},\left\{\frac{x_{1}}{0.8}, \frac{x_{2}}{0.7}, \frac{x_{3}}{0.8}\right\}\right),\left(e_{2},\left\{\frac{x_{1}}{0.6}, \frac{x_{2}}{0.7}, \frac{x_{3}}{0.2}\right\}\right)\right\}$.

We define a 0.3 -cut level utility function $\mu_{0.3}: U \rightarrow \mathbf{R}$ as $\mu_{0.3}\left(x_{1}\right)=5$, $\mu_{0.3}\left(x_{2}\right)=-2$ and $\mu_{0.3}\left(x_{3}\right)=3$.

Let $\left(\alpha_{1}, \alpha_{2}, \alpha_{3}\right)=(1,4,5)$, then $(1,4,5)+0.3 \overrightarrow{(F, A)}=\{(2,5,6),(2,5,5)\}$. Then $0.3\left(\overrightarrow{G, A)}=\{(2,5,6),(2,5,5)\}\right.$. So, $0.3 \overrightarrow{G_{A}}=(2,5,5)$. Here, $\mathrm{h}=0.8$

$$
\sigma\left(0.3 \overrightarrow{\left(G_{F}, A\right)}\right)=\frac{1}{\sqrt{2}} .
$$

Again, $0.3 \overrightarrow{G_{A}^{0.8}}=\left(\frac{2}{0.8}, \frac{5}{0.8}, \frac{5}{0.8}\right)$ and hence, $\sigma\left(0.3 \overrightarrow{\left(G_{F}, A\right)_{0.8}}\right)=\frac{1}{0.8} \times \frac{1}{\sqrt{2}}$.

Thus, calculating we have $0.3 F S C V \overrightarrow{\left(G_{F}, A\right)_{0.8}}=0.8 \times 0.3 F S C V \overrightarrow{\left(G_{F}, A\right)}$.

Remark 3.1. Scaling on an $\alpha$-cut generated fuzzy soft set may not generate distinct $\alpha$-cut generated fuzzy soft set.

The above remark can be verified from the following example.

Example 3.8. Let us consider Example 3.5., where, $(F, A)=\left\{\left(e_{1},\left\{\frac{x_{1}}{0.4}, \frac{x_{2}}{0.8}, \frac{x_{3}}{0.5}\right\}\right),\left(e_{2},\left\{\frac{x_{1}}{0.6}, \frac{x_{2}}{0.2}, \frac{x_{3}}{0.2}\right\}\right)\right\}$.

Then, we have $\left(G_{F}, A\right)_{0.3}=\left\{\left(e_{1},\left\{\frac{x_{1}}{0}, \frac{x_{2}}{0.3}, \frac{x_{3}}{0.3}\right\}\right),\left(e_{2},\left\{\frac{x_{1}}{0}, \frac{x_{2}}{0.2}, \frac{x_{3}}{0.2}\right\}\right)\right.$.

Now, $\frac{(1,4,5)+0.3 \overrightarrow{(F, A)}}{0.8}=\{(2.5,6.25,7.5),(2.5,6.25,6.25)\}$.

Then, for $(2.5,6.25,7.5)$, membership value of $x_{1}=0$, membership value of $x_{2}=0.3$, membership value of $x_{3}=0.3$.

Again, for $(2.5,6.25,6.25)$, membership value of $x_{1}=0$, membership value of $x_{2}=0.2$, membership value of $x_{3}=0.2$.

Thus, $\left(G_{F}, A\right)_{0.3}^{0.8}=\left\{\left(e_{1},\left\{\frac{x_{1}}{0}, \frac{x_{2}}{0.3}, \frac{x_{3}}{0.3}\right\}\right),\left(e_{2},\left\{\frac{x_{1}}{0}, \frac{x_{2}}{0.2}, \frac{x_{3}}{0.2}\right\}\right)\right.$.

This example shows that utility matters more than scaling to generate fuzzy soft set with $\alpha$-cut . 
650 S. Acharjee, D.J. Sarma, R.A. Hanneman, J.N. Mordeson and D.S. Malik

\subsection{Fuzzy soft covariance with $\alpha$-cut}

\section{Definition 3.6.}

(i) Let us consider a universe $U$ with the set of attributes $E$. Let $(F, A)$ and $(G, B)$ be two fuzzy soft sets, where $A, B \subseteq E,|A|=n>|B|=m$. We extend $B$ to $C=B \cup\left\{f_{m+1}, f_{m+2}, \ldots, f_{n}\right\}$ such that $G_{i}\left(f_{k}\right)\left(x_{i}\right)=0$ $\forall k \in\{m+1, m+2, \ldots, n\}$. Then, $\alpha$-cut level fuzzy soft covariance of $(F, A)$ and $(G, B)$ is denoted by $\alpha F S \operatorname{Cov}\left(\overrightarrow{(F, A)}_{\overrightarrow{(G, B)}} \overrightarrow{A|>| B \mid}\right)$ and it is defined as $\alpha F S \operatorname{Cov}(\overrightarrow{(F, A)}, \overrightarrow{(G, B)}|A|>|B|)=\frac{1}{n}\left\{\left\|\Delta_{1}\right\|^{2}+\left\|\Delta_{2}\right\|^{2}+\ldots+\|\right.$ $\left.\Delta_{n} \|^{2}\right\}$, where $\Delta_{j}=\left(\min \left\{\alpha F_{1}\left(e_{j}\right), \alpha G_{1}\left(f_{j}\right)\right\}, \min \left\{\alpha F_{2}\left(e_{j}\right), \alpha G_{2}\left(f_{j}\right)\right\}, \ldots\right.$, $\left.\min \left\{\alpha F_{i}\left(e_{j}\right), \alpha G_{i}\left(f_{j}\right)\right\}, \ldots\right)$, and $e_{j} \in A, f_{j} \in C, i \in \Delta, j \in I$.

The attributes $f_{m+1}, f_{m+2}, \ldots, f_{n}$ with $G_{i}\left(f_{k}\right)\left(x_{j}\right)=0 \forall k \in\{m+1, m+$ $2, \ldots, n\}, i \in \Delta$ are called fuzzy soft statistical dummy attributes for $B$ relative to $A$.

(ii) Let us consider a universe $U$ with the set of attributes $E$. Let $(F, A)$ and $(G, B)$ be two fuzzy soft sets, where $A, B \subseteq E,|A|=n=|B|$. Then, $\alpha$-cut level fuzzy soft covariance of $(F, A)$ and $(G, B)$ is denoted by $\alpha F \operatorname{Cov}\left(\overrightarrow{(F, A)}_{(\overrightarrow{G, B)}} \overrightarrow{A|=| B \mid}_{)}\right)$and it is defined as

$\alpha F S \operatorname{Cov}\left(\overrightarrow{(F, A)}, \overrightarrow{(G, B)}_{|A|=|B|}\right)=\frac{1}{n}\left\{\left\|\Delta_{1}\right\|^{2}+\left\|\Delta_{2}\right\|^{2}+\ldots+\left\|\Delta_{n}\right\|^{2}\right\}$, where

$\Delta_{j}=\left(\min \left\{\alpha F_{1}\left(e_{j}\right), \alpha G_{1}\left(f_{j}\right)\right\}, \min \left\{\alpha F_{2}\left(e_{j}\right), \alpha G_{2}\left(f_{j}\right)\right\}, \ldots\right.$, $\left.\min \left\{\alpha F_{i}\left(e_{j}\right), \alpha G_{i}\left(f_{j}\right)\right\}, \ldots\right)$, and $e_{j} \in A, f_{j} \in B, i \in \Delta, j \in I$.

The above definition can be redefined if $A=B$. In this case $e_{j}=f_{j} \forall j \in I$.

Definition 3.7. (i) Let us consider a universe $U$ with the set of attributes $E$. Let $(F, A)$ and $(G, B)$ be two fuzzy soft sets, where $A, B \subseteq$ $E,|A|=n>|B|=m$. We extend $B$ to $C=B \cup\left\{f_{m+1}, f_{m+2}, \ldots, f_{n}\right\}$ such that $G_{i}\left(f_{k}\right)\left(x_{i}\right)=0 \forall k \in\{m+1, m+2, \ldots, n\}$. Then, $(F, A)$ and $(G, B)$ are said to be $\epsilon_{\alpha}$ approximation independent fuzzy soft sets if $\Delta_{j}=$ $\left(\min \left\{\alpha F_{1}\left(e_{j}\right), \alpha G_{1}\left(f_{j}\right)\right\}, \min \left\{\alpha F_{2}\left(e_{j}\right), \alpha G_{2}\left(f_{j}\right)\right\}, \ldots\right.$,

$\left.\min \left\{\alpha F_{i}\left(e_{j}\right), \alpha G_{i}\left(f_{j}\right)\right\}, \ldots\right)=0$, where $0=(0,0, \ldots, 0, \ldots), e_{j} \in A$ and $f_{j} \in C, i \in \Delta, \forall j \in I$.

(ii) Let us consider a universe $U$ with the set of attributes $E$. Let $(F, A)$ and $(G, B)$ be two fuzzy soft sets, where $A, B \subseteq E,|A|=n=|B|$. Then, 
$(F, A)$ and $(G, B)$ are said to be $\epsilon_{\alpha^{-}}$approximation independent fuzzy soft sets if $\Delta_{j}=\left(\min \left\{\alpha F_{1}\left(e_{j}\right), \alpha G_{1}\left(f_{j}\right)\right\}, \min \left\{\alpha F_{2}\left(e_{j}\right), \alpha G_{2}\left(f_{j}\right)\right\}, \ldots\right.$,

$\left.\min \left\{\alpha F_{i}\left(e_{j}\right), \alpha G_{i}\left(f_{j}\right)\right\}, \ldots\right)=0$, where $0=(0,0, \ldots, 0, \ldots), e_{j} \in A$ and $f_{j} \in B, i \in \Delta, \forall j \in I$.

Theorem 3.5. (i) Let us consider a universe $U$ with the set of attributes $E$. Let $(F, A)$ and $(G, B)$ be two fuzzy soft sets, where $A, B \subseteq E,|A|$ $=n>|B|=m$. Then, $\alpha F S \operatorname{Cov}(\overrightarrow{(F, A)}, \overrightarrow{(G, B)}|A|>|B|)=0 \Leftrightarrow(F, A)$ and $(G, B)$ are $\epsilon_{\alpha}$-approximation independent fuzzy soft sets.

(ii) Let us consider a universe $U$ with the set of attributes $E$. Let $(F, A)$ and $(G, B)$ be two fuzzy soft sets, where $A, B \subseteq E,|A|=n=|B|$. Then, $\alpha F S \operatorname{Cov}\left(\overrightarrow{(F, A)}, \overrightarrow{(G, B)}_{|A|=|B|}\right)=0 \Leftrightarrow(F, A)$ and $(G, B)$ are $\epsilon_{\alpha^{-}}$ approximation independent fuzzy soft sets.

Proof: (i) $\alpha F S \operatorname{Cov}\left(\overrightarrow{(F, A)}_{\overrightarrow{(G, B)}} \overrightarrow{|A|>|B|}_{\mid}\right)=0$

$\Leftrightarrow \frac{1}{n}\left\{\left\|\Delta_{1}\right\|^{2}+\left\|\Delta_{2}\right\|^{2}+\ldots+\left\|\Delta_{n}\right\|^{2}\right\}=0$

$\Leftrightarrow\left\|\Delta_{j}\right\|^{2}=0 \forall j \in I$

$\Leftrightarrow\left\|\Delta_{j}\right\|=0 \forall j \in I$

$\Leftrightarrow \Delta_{j}=(0,0, \ldots, 0, \ldots) \forall j \in I$

$\Leftrightarrow(F, A)$ and $(G, B)$ are $\epsilon_{\alpha}$-approximation independent fuzzy soft sets.

(ii) Proof can be done following the above steps.

\section{Fuzzy soft attribute correlation coefficient and $\alpha$-cut}

In Definition 3.2, we defined $\alpha \overrightarrow{F\left(e_{i}\right)}$. Now, we consider the following example.

Example 4.1. Let $U=\left\{x_{1}, x_{2}, x_{3}, x_{4}, x_{5}\right\}$ be a universe and $E=\left\{e_{1}, e_{2}, e_{3}, e_{4}\right\}$ be a set of attributes. We consider $A=\left\{e_{1}, e_{2}, e_{3}\right\}$ and define a fuzzy soft set as

$(F, A)=\left\{\left(e_{1},\left\{\frac{x_{1}}{0.1}, \frac{x_{2}}{0}, \frac{x_{3}}{0.5}, \frac{x_{4}}{0.9}, \frac{x_{5}}{0}\right\}\right),\left(e_{2},\left\{\frac{x_{1}}{0}, \frac{x_{2}}{0.8}, \frac{x_{3}}{0.4}, \frac{x_{4}}{0.4}, \frac{x_{5}}{0}\right\}\right)\right.$,

$\left.\left(e_{3},\left\{\frac{x_{1}}{0.2}, \frac{x_{2}}{0}, \frac{x_{3}}{0.8}, \frac{x_{4}}{0.9}, \frac{x_{5}}{0}\right\}\right)\right\} ;$ then $0.1 \overrightarrow{F\left(e_{1}\right)}=(1,0,1,1,0), 0.3 \overrightarrow{F\left(e_{1}\right)}$

$=(0,0,1,1,0), 0.5 \overrightarrow{F\left(e_{3}\right)}=(0,0,1,1,0)$, etc. 
652 S. Acharjee, D.J. Sarma, R.A. Hanneman, J.N. Mordeson and D.S. Malik

Definition 4.1. Let $(F, A)$ be a fuzzy soft set with at least two attributes $e_{1}$ and $e_{2}$, then fuzzy soft attribute correlation coefficient of $\alpha \overrightarrow{F\left(e_{1}\right)}$ and $\alpha \overrightarrow{F\left(e_{2}\right)}$ at $\alpha$-cut level is defined as follows:

$$
\begin{gathered}
F S A C C\left(\alpha \overrightarrow{F\left(e_{1}\right)}, \alpha \overrightarrow{F\left(e_{2}\right)}\right)=\frac{\sum_{i \in I} \Delta_{i}}{\left\|\alpha \overrightarrow{F\left(e_{1}\right)}\right\| \cdot\left\|\alpha \overrightarrow{F\left(e_{2}\right)}\right\|} \text {; where } \\
\Delta_{i}=\min \left\{\alpha F_{i}\left(e_{1}\right), \alpha F_{i}\left(e_{2}\right)\right\},\left\|\alpha \overrightarrow{F\left(e_{j}\right)}\right\|=\sqrt{\left\langle\alpha \overrightarrow{F\left(e_{j}\right)}, \alpha \overrightarrow{F\left(e_{j}\right)}\right\rangle}= \\
\sqrt{\sum_{i \in I}\left(\alpha F_{i}\left(e_{j}\right)\right)^{2}},\left\|\alpha \overrightarrow{F\left(e_{1}\right)}\right\| \neq 0 \text { and }\left\|\alpha \overrightarrow{F\left(e_{2}\right)}\right\| \neq 0 .
\end{gathered}
$$

If $\left\|\alpha \overrightarrow{F\left(e_{1}\right)}\right\|=0$ or $\left\|\alpha \overrightarrow{F\left(e_{2}\right)}\right\|=0$, then $F S A C C\left(\alpha \overrightarrow{F\left(e_{1}\right)}, \alpha \overrightarrow{F\left(e_{2}\right)}\right)$ is not possible. In this case, we shall use notation " $\infty$ " without considering any matter of $\Delta_{i}$.

Example 4.2. Let us consider a universe $U=\left\{x_{1}, x_{2}, x_{3}, x_{4}\right\}$ and a set of attributes $E=\left\{e_{1}, e_{2}, e_{3}, e_{4}\right\}$. Let $A=\left\{e_{1}, e_{2}, e_{3}\right\}$. We define a fuzzy soft set $(F, A)=\left\{\left(e_{1},\left\{\frac{x_{1}}{0.1}, \frac{x_{2}}{0.7}, \frac{x_{3}}{0.2}, \frac{x_{4}}{0}\right\}\right),\left(e_{2},\left\{\frac{x_{1}}{0.2}, \frac{x_{2}}{0}, \frac{x_{3}}{0.4}, \frac{x_{4}}{0.5}\right\}\right)\right.$,

$\left.\left(e_{3},\left\{\frac{x_{1}}{0}, \frac{x_{2}}{0.3}, \frac{x_{3}}{0.5}, \frac{x_{4}}{0}\right\}\right)\right\}$. Consider $\alpha=0.1$, then $0.1 \overrightarrow{F\left(e_{1}\right)}=(1,1,1,0)$ and $0.1 \overrightarrow{F\left(e_{2}\right)}=(1,0,1,1)$. Then $\Delta_{1}=\min \{1,1\}=1, \Delta_{2}=\min \{1,0\}=0$, $\Delta_{3}=\min \{1,1\}=1$ and $\Delta_{4}=\min \{0,1\}=0$. Then, $F S A C C\left(0.1 \overrightarrow{F\left(e_{1}\right)}, 0.1 \overrightarrow{F\left(e_{2}\right)}\right)=0.67$.

Theorem 4.1. If $(F, A)$ be any fuzzy soft set with at least two attributes $e_{1}$ and $e_{2}$ over a universe $U$, then $0 \leq F S A C C\left(\alpha \overrightarrow{F\left(e_{1}\right)}, \alpha \overrightarrow{F\left(e_{2}\right)}\right) \leq 1$.

Proof. We know, $\sum_{i \in I} \Delta_{i}=\sum_{i \in I} \min \left\{\alpha F_{i}\left(e_{1}\right), \alpha F_{i}\left(e_{2}\right)\right\} \leq \sum_{i \in I}\left(\alpha F_{i}\left(e_{1}\right) \cdot \alpha F_{i}\left(e_{2}\right)\right)$
$\leq \sqrt{\sum_{i \in I}\left(\alpha F_{i}\left(e_{1}\right)\right)^{2}} \cdot \sqrt{\sum_{i \in I}\left(\alpha F_{i}\left(e_{2}\right)\right)^{2}}=\left\|\alpha \overrightarrow{F\left(e_{1}\right)}\right\| \cdot\left\|\alpha \overrightarrow{F\left(e_{2}\right)}\right\|$.
$\quad$ So, $\frac{\sum_{i \in I} \Delta_{i}}{\left\|\alpha \overrightarrow{F\left(e_{1}\right)}\right\| \cdot \| \alpha \overrightarrow{F\left(e_{2}\right) \|}} \leq 1$, which implies $F S A C C\left(\alpha \overrightarrow{F\left(e_{1}\right)}, \alpha \overrightarrow{F\left(e_{2}\right)}\right) \leq 1$.

Also, $\sum_{i \in I} \Delta_{i} \geq 0$ and $\left\|\alpha \overrightarrow{F\left(e_{1}\right)}\right\| .\left\|\alpha \overrightarrow{F\left(e_{2}\right)}\right\|>0$ which implies $0 \leq F S A C C\left(\alpha \overrightarrow{F\left(e_{1}\right)}, \alpha \overrightarrow{F\left(e_{2}\right)}\right)$. 
Hence, $0 \leq F S A C C\left(\alpha \overrightarrow{F\left(e_{1}\right)}, \alpha \overrightarrow{F\left(e_{2}\right)}\right) \leq 1$.

Theorem 4.2. Let $(F, A)$ be a fuzzy soft set with at least two attributes $e_{1}$ and $e_{2}$, then $F S A C C\left(\alpha \overrightarrow{F\left(e_{1}\right)}, \alpha \overrightarrow{F\left(e_{2}\right)}\right)=F S A C C\left(\alpha \overrightarrow{F\left(e_{2}\right)}, \alpha \overrightarrow{F\left(e_{1}\right)}\right)$.

Proof. Proof can be obtained from Definition 4.1.

Theorem 4.3. If $F\left(e_{1}\right), G\left(e_{1}\right)$ and $H\left(e_{1}\right)$ are fuzzy sets of fuzzy soft sets $(F, A),(G, A)$ and $(H, A)$ respectively over $U$ such that $F\left(e_{1}\right) \subseteq G\left(e_{1}\right) \subseteq$ $H\left(e_{1}\right)$ and $e_{1} \in A$, then;

(i) $F S A C C\left(\alpha \overrightarrow{F\left(e_{1}\right)}, \alpha \overrightarrow{H\left(e_{1}\right)}\right) \leq F S A C C\left(\alpha \overrightarrow{F\left(e_{1}\right)}, \alpha \overrightarrow{G\left(e_{1}\right)}\right)$

(ii) $F S A C C\left(\alpha \overrightarrow{F\left(e_{1}\right)}, \alpha \overrightarrow{H\left(e_{1}\right)}\right) \leq F S A C C\left(\alpha \overrightarrow{G\left(e_{1}\right)}, \alpha \overrightarrow{H\left(e_{1}\right)}\right)$

(iii) $F S A C C\left(\alpha \overrightarrow{F\left(e_{1}\right)}, \alpha \overrightarrow{G\left(e_{1}\right)}\right) \leq F S A C C\left(\alpha \overrightarrow{H\left(e_{1}\right)}, \alpha \overrightarrow{G\left(e_{1}\right)}\right)$ if $\left(\left\|\alpha \overrightarrow{F\left(e_{1}\right)}\right\| \cdot\left\|\alpha \overrightarrow{H\left(e_{1}\right)}\right\|-\left\|\alpha \overrightarrow{G\left(e_{1}\right)}\right\|^{2}\right) \leq 0$

Proof. (i) Since $F\left(e_{1}\right) \subseteq G\left(e_{1}\right) \subseteq H\left(e_{1}\right)$, so $F_{j}\left(e_{1}\right)\left(x_{j}\right) \leq G_{j}\left(e_{1}\right)\left(x_{j}\right) \leq H_{j}\left(e_{1}\right)\left(x_{j}\right)$. Thus, $\Delta_{i}=\min \left\{\alpha F_{i}\left(e_{1}\right), \alpha H_{i}\left(e_{1}\right)\right\}=$ $\alpha F_{i}\left(e_{1}\right)$

and $\Delta_{i}^{\prime}=\min \left\{\alpha F_{i}\left(e_{1}\right), \alpha G_{i}\left(e_{1}\right)\right\}=\alpha F_{i}\left(e_{1}\right)$. Thus, $\Delta_{i}=\Delta_{i}^{\prime}$.

Now, FSACC $\left(\alpha \overrightarrow{F\left(e_{1}\right)}, \alpha \overrightarrow{H\left(e_{1}\right)}\right)-F S A C C\left(\alpha \overrightarrow{F\left(e_{1}\right)}, \alpha \overrightarrow{G\left(e_{1}\right)}\right)$

$$
\begin{aligned}
& =\frac{\sum_{i \in I} \Delta_{i}}{\left\|\alpha \overrightarrow{F\left(e_{1}\right)}\right\| \cdot\left\|\alpha \overrightarrow{H\left(e_{1}\right)}\right\|}-\frac{\sum_{i \in I} \Delta_{i}^{\prime}}{\left\|\alpha \overrightarrow{F\left(e_{1}\right)}\right\| \cdot\left\|\alpha \overrightarrow{G\left(e_{1}\right)}\right\|} \\
& =\frac{\sum_{i \in I} \Delta_{i}}{\left\|\alpha \overrightarrow{F\left(e_{1}\right)}\right\|} \cdot\left\{\frac{1}{\left\|\alpha \overrightarrow{H\left(e_{1}\right)}\right\|}-\frac{1}{\left\|\alpha \overrightarrow{G\left(e_{1}\right)}\right\|}\right\} \ldots \ldots .
\end{aligned}
$$

Since, $G\left(e_{1}\right) \subseteq H\left(e_{1}\right)$ and $G\left(e_{1}\right), H\left(e_{1}\right)$ are fuzzy sets, so $\alpha G_{i}\left(e_{1}\right) \leq$ $\alpha H_{i}\left(e_{1}\right) \Rightarrow \sum_{i \in I}\left\{\alpha G_{i}\left(e_{1}\right)\right\}^{2} \leq \sum_{i \in I}\left\{\alpha H_{i}\left(e_{1}\right)\right\}^{2}$

$\Rightarrow\left\|\alpha \overrightarrow{G\left(e_{1}\right)}\right\| \leq\left\|\alpha \overrightarrow{H\left(e_{1}\right)}\right\| \Rightarrow\left\{\frac{1}{\left\|\alpha \overrightarrow{H\left(e_{1}\right)}\right\|}-\frac{1}{\left\|\alpha \overrightarrow{G\left(e_{1}\right)}\right\|}\right\} \leq 0 \ldots$

So, (3.1) and (3.2.) implies $F S A C C\left(\alpha \overrightarrow{F\left(e_{1}\right)}, \alpha \overrightarrow{H\left(e_{1}\right)}\right) \leq F S A C C\left(\alpha \overrightarrow{F\left(e_{1}\right)}, \alpha \overrightarrow{G\left(e_{1}\right)}\right)$. 
654 S. Acharjee, D.J. Sarma, R.A. Hanneman, J.N. Mordeson and D.S. Malik

(ii) Since $F\left(e_{1}\right) \subseteq G\left(e_{1}\right) \subseteq H\left(e_{1}\right)$, so

$F_{j}\left(e_{1}\right)\left(x_{j}\right) \leq G_{j}\left(e_{1}\right)\left(x_{j}\right) \leq H_{j}\left(e_{1}\right)\left(x_{j}\right)$. Thus, $\Delta_{i}=\min \left\{\alpha F_{i}\left(e_{1}\right), \alpha H_{i}\left(e_{1}\right)\right\}=$ $\alpha F_{i}\left(e_{1}\right)$ and $\Delta_{i}^{\prime}=\min \left\{\alpha G_{i}\left(e_{1}\right), \alpha H_{i}\left(e_{1}\right)\right\}=\alpha G_{i}\left(e_{1}\right)$.

Now, FSACC $\left(\alpha \overrightarrow{F\left(e_{1}\right)}, \alpha \overrightarrow{H\left(e_{1}\right)}\right)-F S A C C\left(\alpha \overrightarrow{G\left(e_{1}\right)}, \alpha \overrightarrow{H\left(e_{1}\right)}\right)$

$$
\begin{aligned}
& =\frac{\sum_{i \in I} \Delta_{i}}{\left\|\alpha \overrightarrow{F\left(e_{1}\right)}\right\| \cdot\left\|\alpha \overrightarrow{H\left(e_{1}\right)}\right\|}-\frac{\sum_{i \in I} \Delta_{i}^{\prime}}{\left\|\alpha \overrightarrow{G\left(e_{1}\right)}\right\| \cdot\left\|\alpha \overrightarrow{H\left(e_{1}\right)}\right\|} . \\
& =\frac{1}{\left\|\alpha \overrightarrow{H\left(e_{1}\right)}\right\|} \cdot\left\{\frac{\sum_{i \in I}\left\{\alpha F_{i}\left(e_{1}\right)\right\}^{2}}{\left\|\alpha \overrightarrow{F\left(e_{1}\right)}\right\|}-\frac{\sum_{i \in I}\left\{\alpha G_{i}\left(e_{1}\right)\right\}^{2}}{\left\|\alpha \overrightarrow{G\left(e_{1}\right)}\right\|}\right\} \\
& =\frac{1}{\left\|\alpha \overrightarrow{H\left(e_{1}\right)}\right\|} \cdot\left\{\frac{\left\|\alpha \overrightarrow{F\left(e_{1}\right)}\right\|^{2}}{\left\|\alpha \overrightarrow{F\left(e_{1}\right)}\right\|}-\frac{\left\|\alpha \overrightarrow{G\left(e_{1}\right)}\right\|^{2}}{\left\|\alpha \overrightarrow{G\left(e_{1}\right)}\right\|}\right\} \\
& =\frac{1}{\left\|\alpha \overrightarrow{H\left(e_{1}\right)}\right\|} \cdot\left\{\left\|\alpha \overrightarrow{F\left(e_{1}\right)}\right\|-\left\|\alpha \overrightarrow{G\left(e_{1}\right)}\right\|\right\} \leq 0
\end{aligned}
$$

Hence, $F S A C C\left(\alpha \overrightarrow{F\left(e_{1}\right)}, \alpha \overrightarrow{H\left(e_{1}\right)}\right) \leq F S A C C\left(\alpha \overrightarrow{G\left(e_{1}\right)}, \alpha \overrightarrow{H\left(e_{1}\right)}\right)$.

(iii) Proof can be done by following the above steps.

Now, we state the following theorem without proof.

Theorem 4.4. $F S A C C\left(\alpha \overrightarrow{F\left(e_{1}\right)}, \alpha \overrightarrow{F\left(e_{2}\right)}\right)=1 \Leftrightarrow \alpha \overrightarrow{F\left(e_{1}\right)}=\alpha \overrightarrow{F\left(e_{2}\right)}$

\section{Application to human trafficking}

In this section, we do analysis of fuzzy soft attribute correlation coefficient $(F S A C C$ ) between mean of vulnerability and mean of government responses of the following regions: Asia Pacific, Europe, Russia and Eurasia, Sub-Saharan Africa, The Middle East and North Africa, and The Americas. For the purpose of region-wise data related to vulnerability and government responses, we consider region-wise data of "The Global Slavery Index 2016" $[4]$.

According to "The Global Slavery index 2016" [4], twenty four vulnerability variables are clustered in following four dimensions. 
Dimension 1: Civil and political protections: Confidence in judicial system, political stability, weapons access, discrimination (sexuality), displaced persons, global slavery index government response values, political rights measure.

Dimension 2: Social, health and economic rights: Financial inclusion (borrowed any money), financial inclusion (received wages), cell phone subscription, safety net, undernourishment, tuberculosis, water improved access.

Dimension 3: Personal security: Financial inclusion (availability of emergency funds), violent crime, women's physical security, GINI coefficient, discrimination ( intellectual disability), discrimination (immigrants), discrimination( minorities).

Dimension 4: Refugee populations and conflict: Impact of terrorism, internal conflict, refugees resident.

Now, we denote two sets of parameters $P$ and $Q$ related to vulnerability and government responses respectively.

Let $P=\left\{e_{1}, e_{2}, e_{3}, e_{4}\right\}, Q=\left\{f_{1}, f_{2}, f_{3}, f_{4}, f_{5}\right\}$.

We consider the following notions for the attributes of vulnerability and government responses.

$$
\begin{aligned}
& e_{1}=\text { Civil and political protection } ; \\
& e_{2}=\text { Social, health, and economic rights; } \\
& e_{3}=\text { Personal security } \\
& e_{4}=\text { Refugees and conflict } \\
& f_{1}=\text { Survivors supported } \\
& f_{2}=\text { Criminal justice } \\
& f_{3}=\text { Coordination and accountability; }
\end{aligned}
$$


656 S. Acharjee, D.J. Sarma, R.A. Hanneman, J.N. Mordeson and D.S. Malik

$$
\begin{aligned}
& f_{4}=\text { Addressing risk } \\
& f_{5}=\text { Government and business } .
\end{aligned}
$$

Let $A=\left\{g_{1}, g_{2}, g_{3}\right\}$, where $g_{1}=$ mean of vulnerability, $g_{2}=$ mean of government responses and $g_{3}=100$ - mean of vulnerability.

Throughout this article, $F S A C C_{\alpha}($ Asia Pacific) means $F S A C C\left(\alpha \overrightarrow{F\left(g_{1}\right)}, \alpha \overrightarrow{F\left(g_{2}\right)}\right)$ for Asia Pacific and so on for other five regions. Similarly, we shall denote $F S A C C_{\alpha}^{C}$ (Asia Pacific) means $F S A C C\left(\alpha \overrightarrow{F\left(g_{2}\right)}, \alpha \overrightarrow{F\left(g_{3}\right)}\right)$ for Asia Pacific and so on for other five regions.

Let $X$ be the set of 167 countries, which are considered in "The Global Slavery Index 2016" [4] and $U_{i}, i \in\{1,2,3,4,5,6\}$ be the regions Asia Pacific, Russia and Eurasia, The Middle East and North Africa, The Americas, Europe, and Sub-Saharan Africa respectively. We define fuzzy soft sets $F_{U_{i}}: A \rightarrow[0,1]^{U_{i}} \forall i \in\{1,2,3,4,5,6\}$ with the following membership values.

\begin{tabular}{|c|c|}
\hline fuzzy membership $(\alpha)$ & conditions \\
\hline 0 & if mean value of $x_{i j} \leq 0$ \\
0.1 & if $0<$ mean value of $x_{i j} \leq 10$ \\
0.2 & if $10<$ mean value of $x_{i j} \leq 20$ \\
0.3 & if $20<$ mean value of $x_{i j} \leq 30$ \\
0.4 & if $30<$ mean value of $x_{i j} \leq 40$ \\
0.5 & if $40<$ mean value of $x_{i j} \leq 50$ \\
0.6 & if $50<$ mean value of $x_{i j} \leq 60$ \\
0.7 & if $60<$ mean value of $x_{i j} \leq 70$ \\
0.8 & if $70<$ mean value of $x_{i j} \leq 80$ \\
0.9 & if $80<$ mean value of $x_{i j} \leq 90$ \\
1 & if $90<$ mean value of $x_{i j} \leq 100$ \\
\hline
\end{tabular}

Table 4

Here, $x_{i j}$ indicates the country which is in the $i^{\text {th }}$ place with alphabetic order in the region $U_{j}$ of "The Global Slavery Index 2016" [4], for $j \in\{1,2,3,4,5,6\}$. 


\section{(i) Asia Pacific}

We shall use following abbreviations:

$H S C=$ Hong Kong, SAR China

$P N G=$ Papua New Guinea

According to "The Global Slavery Index 2016"([4], p. 55); government responses of Afghanistan were unknown for $f_{1}, f_{2}, f_{3}, f_{4}$ and $f_{5}$, hence we shall consider 0 for Afghanistan. It is because of either government of Afghanistan was unable to take action against human trafficking in Afghanistan or government was not interested to take part in worldwide research survey related to human trafficking. Moreover, many internal causes may be present including terrorism threat, etc.

Similar representations will be considered for similar cases. 
658 S. Acharjee, D.J. Sarma, R.A. Hanneman, J.N. Mordeson and D.S. Malik

\begin{tabular}{|c|c|c|c|c|c|c|c|c|c|c|c|c|c|}
\hline Name & $e_{1}$ & $e_{2}$ & $e_{3}$ & $e_{4}$ & Mean & FMV & $f_{1}$ & $f_{2}$ & $f_{3}$ & $f_{4}$ & $f_{5}$ & Mean & FMV \\
\hline Afghanistan* & 83.00 & 47.39 & 53.31 & 84.55 & 67.06 & 0.70 & 0.00 & 0.00 & 0.00 & 0.00 & 0.00 & 0.00 & 0.00 \\
\hline Australia & 15.14 & 19.85 & 17.45 & 35.49 & 21.98 & 0.30 & 64.44 & 81.85 & 56.25 & 69.05 & 25.00 & 59.32 & 0.60 \\
\hline Bangladesh & 46.78 & 46.04 & 33.63 & 50.02 & 44.12 & 0.50 & 39.44 & 60.37 & 68.75 & 59.52 & 0.00 & 45.62 & 0.50 \\
\hline Brunei & 60.78 & 30.99 & 99.99 & 63.93 & 63.92 & 0.70 & 7.41 & 35.74 & 12.50 & 30.95 & 0.00 & 17.32 & 0.20 \\
\hline Cambodia & 53.68 & 42.96 & 57.40 & 12.00 & 41.51 & 0.50 & 25.19 & 33.33 & 37.50 & 64.29 & 0.00 & 32.06 & 0.40 \\
\hline China & 55.12 & 26.90 & 43.84 & 52.78 & 44.66 & 0.50 & 35.56 & 23.70 & 31.25 & 52.38 & 0.00 & 28.58 & 0.30 \\
\hline $\mathrm{HSC}$ & 42.28 & 17.55 & 21.44 & 35.65 & 29.23 & 0.30 & 5.93 & 5.19 & 0.00 & 30.95 & 0.00 & 8.41 & 0.10 \\
\hline India & 37.07 & 36.68 & 43.88 & 87.78 & 51.35 & 0.60 & 44.07 & 45.00 & 43.75 & 45.24 & 0.00 & 35.61 & 0.40 \\
\hline Indonesia & 39.15 & 43.35 & 50.38 & 36.01 & 42.22 & 0.50 & 37.59 & 40.56 & 50.00 & 54.76 & 0.00 & 36.58 & 0.40 \\
\hline Japan & 25.23 & 19.09 & 22.16 & 19.13 & 21.40 & 0.30 & 42.59 & 19.44 & 18.75 & 45.24 & 0.00 & 25.20 & 0.30 \\
\hline Laos & 56.64 & 34.01 & 53.98 & 1.19 & 36.45 & 0.40 & 28.70 & 26.48 & 31.25 & 50.00 & 0.00 & 27.29 & 0.30 \\
\hline Malaysia & 34.90 & 32.43 & 46.39 & 40.33 & 38.51 & 0.40 & 36.48 & 51.48 & 31.25 & 35.71 & 0.00 & 30.98 & 0.40 \\
\hline Mongolia & 39.22 & 36.74 & 40.87 & 3.54 & 30.09 & 0.40 & 27.78 & 31.67 & 31.25 & 47.62 & 0.00 & 27.66 & 0.30 \\
\hline Myanmar & 57.81 & 50.11 & 50.53 & 66.99 & 56.36 & 0.60 & 41.11 & 8.89 & 50.00 & 50.00 & 0.00 & 30.00 & 0.30 \\
\hline Nepal & 42.30 & 43.22 & 34.74 & 41.21 & 40.37 & 0.50 & 42.78 & 38.15 & 75.00 & 61.90 & 0.00 & 43.57 & 0.50 \\
\hline New Zealand & 13.31 & 22.24 & 16.09 & 21.51 & 18.29 & 0.20 & 53.70 & 47.96 & 43.75 & 88.10 & 0.00 & 46.70 & 0.50 \\
\hline North Korea & 71.20 & 48.27 & 62.88 & 1.00 & 45.84 & 0.50 & 0.00 & -13.89 & 0.00 & -7.14 & 0.00 & -4.21 & 0.00 \\
\hline Pakistan & 58.40 & 41.98 & 52.70 & 96.79 & 62.47 & 0.70 & 28.52 & 37.04 & 25.00 & 76.19 & 0.00 & 33.35 & 0.40 \\
\hline PNG & 50.12 & 62.85 & 99.99 & 23.10 & 59.01 & 0.60 & 6.48 & 23.70 & 25.00 & 14.29 & 0.00 & 13.89 & 0.20 \\
\hline Philippines & 44.76 & 39.62 & 52.34 & 53.95 & 47.67 & 0.50 & 46.48 & 62.78 & 50.00 & 78.57 & 0.00 & 47.57 & 0.50 \\
\hline Singapore & 29.85 & 22.11 & 20.58 & 1.00 & 18.38 & 0.20 & 36.11 & 22.41 & 0.00 & 42.86 & 0.00 & 20.28 & 0.30 \\
\hline South Korea & 38.20 & 34.64 & 28.98 & 17.32 & 29.79 & 0.30 & 35.93 & 31.85 & 12.50 & 33.33 & 0.00 & 22.72 & 0.30 \\
\hline Sri Lanka & 47.01 & 35.12 & 31.82 & 31.08 & 36.26 & 0.40 & 25.93 & 38.52 & 37.50 & 83.33 & 0.00 & 37.06 & 0.40 \\
\hline Taiwan & 34.91 & 33.38 & 22.34 & 1.76 & 23.10 & 0.30 & 50.56 & 23.62 & 43.75 & 42.86 & 0.00 & 32.16 & 0.40 \\
\hline Thailand & 49.23 & 28.62 & 48.97 & 63.33 & 47.54 & 0.50 & 35.19 & 35.93 & 56.25 & 61.90 & 0.00 & 37.85 & 0.40 \\
\hline Timor-Leste & 38.88 & 48.07 & 68.55 & 1.00 & 39.13 & 0.40 & 25.93 & 25.93 & 0.00 & 40.48 & 0.00 & 18.47 & 0.20 \\
\hline Vietnam & 51.19 & 29.94 & 35.22 & 1.00 & 29.34 & 0.30 & 45.19 & 34.07 & 62.50 & 66.67 & 0.00 & 41.69 & 0.50 \\
\hline
\end{tabular}

Table 5

\begin{tabular}{|c|c|c|c|c|c|c|c|c|c|c|}
\hline$\alpha$ & 0.1 & 0.2 & 0.3 & 0.4 & 0.5 & 0.6 & 0.7 & 0.8 & 0.9 & 1 \\
\hline FSACC(Asia) & 0.9623 & 0.9428 & 0.8292 & 0.6131 & 0.3273 & 0 & $\infty$ & $\infty$ & $\infty$ & $\infty$ \\
\hline
\end{tabular}

Table 6 


\section{(ii) Russia and Eurasia}

\begin{tabular}{|c|c|c|c|c|c|c|c|c|c|c|c|c|c|}
\hline Name & $e_{1}$ & $e_{2}$ & $e_{3}$ & $e_{4}$ & Mean & FMV & $f_{1}$ & $f_{2}$ & $f_{3}$ & $f_{4}$ & $f_{5}$ & Mean & FMV \\
\hline Armenia & 53.66 & 27.38 & 42.63 & 24.90 & 37.14 & 0.40 & 48.33 & 49.81 & 56.25 & 50.00 & 0.00 & 40.88 & 0.50 \\
Azerbaijan & 68.90 & 28.23 & 28.17 & 23.05 & 37.09 & 0.40 & 28.33 & 60.37 & 18.75 & 57.14 & 0.00 & 32.92 & 0.40 \\
Belarus & 58.41 & 20.48 & 35.88 & 23.80 & 34.64 & 0.40 & 35.37 & 26.11 & 43.75 & 52.38 & 0.00 & 31.52 & 0.40 \\
Georgia & 51.00 & 28.43 & 35.95 & 24.42 & 34.95 & 0.40 & 67.59 & 58.70 & 56.25 & 59.52 & 0.00 & 48.41 & 0.50 \\
Kazakhstan & 59.05 & 22.08 & 28.19 & 22.12 & 32.86 & 0.40 & 38.33 & 44.07 & 18.75 & 35.71 & 0.00 & 27.37 & 0.30 \\
Kyrgyzstan & 54.16 & 28.33 & 36.37 & 21.88 & 35.18 & 0.40 & 27.96 & 35.74 & 37.50 & 50.00 & 0.00 & 30.24 & 0.40 \\
Moldova & 47.52 & 28.20 & 38.04 & 14.41 & 32.04 & 0.40 & 55.56 & 57.59 & 50.00 & 64.29 & 0.00 & 45.49 & 0.50 \\
Russia & 57.21 & 18.47 & 40.66 & 57.47 & 43.45 & 0.50 & 21.48 & 28.33 & 12.50 & 61.90 & 0.00 & 24.84 & 0.30 \\
Tajikistan & 62.85 & 37.62 & 41.68 & 27.53 & 42.42 & 0.50 & 41.99 & 34.44 & 25.00 & 54.76 & 0.00 & 31.24 & 0.40 \\
Turkmenistan & 68.14 & 28.65 & 43.34 & 9.22 & 37.34 & 0.40 & 14.81 & 35.74 & 12.50 & 54.76 & 0.00 & 23.56 & 0.30 \\
Ukraine & 61.97 & 21.39 & 35.80 & 43.41 & 40.64 & 0.50 & 62.04 & 47.78 & 12.50 & 61.90 & 0.00 & 36.84 & 0.40 \\
Uzbekistan & 74.62 & 28.35 & 32.09 & 12.14 & 36.80 & 0.40 & 24.26 & 23.70 & 18.75 & 52.38 & 0.00 & 23.82 & 0.30 \\
\hline
\end{tabular}

Table 7

\begin{tabular}{|c|c|c|c|c|c|c|c|c|c|c|}
\hline$\alpha$ & 0.1 & 0.2 & 0.3 & 0.4 & 0.5 & 0.6 & 0.7 & 0.8 & 0.9 & 1 \\
\hline FSACC(Russia and Eurasia) & 1 & 1 & 1 & 0.8165 & 0 & $\infty$ & $\infty$ & $\infty$ & $\infty$ & $\infty$ \\
\hline
\end{tabular}

Table 8 
660 S. Acharjee, D.J. Sarma, R.A. Hanneman, J.N. Mordeson and D.S. Malik

(iii) The Middle East and North Africa

Throughout this paper, we shall use "MENA" as an abbreviation to "The Middle East and North Africa"

\begin{tabular}{|c|c|c|c|c|c|c|c|c|c|c|c|c|c|}
\hline Name & $e_{1}$ & $e_{2}$ & $e_{3}$ & $e_{4}$ & Mean & FMV & $f_{1}$ & $f_{2}$ & $f_{3}$ & $f_{4}$ & $f_{5}$ & Mean & FMV \\
\hline Algeria & 51.03 & 28.10 & 47.02 & 51.46 & 44.40 & 0.50 & 28.52 & 24.07 & 25.00 & 42.86 & 0.00 & 24.09 & 0.30 \\
Bahrain & 54.41 & 33.14 & 41.45 & 31.26 & 40.06 & 0.50 & 36.67 & 36.67 & 25.00 & 35.71 & 0.00 & 26.81 & 0.30 \\
Egypt & 51.25 & 27.90 & 62.96 & 54.85 & 49.24 & 0.50 & 35.74 & 32.04 & 50.00 & 52.38 & 0.00 & 34.03 & 0.40 \\
Iran & 70.71 & 32.99 & 48.60 & 51.44 & 50.93 & 0.60 & 0.00 & 7.41 & 0.00 & 16.67 & 0.00 & 4.82 & 0.10 \\
Iraq* & 71.22 & 44.91 & 58.04 & 81.13 & 63.82 & 0.70 & 0.00 & 0.00 & 0.00 & 0.00 & 0.00 & 0.00 & 0.00 \\
Israel & 33.66 & 23.67 & 38.28 & 51.85 & 36.87 & 0.40 & 49.81 & 47.96 & 50.00 & 61.90 & 0.00 & 41.93 & 0.50 \\
Jordan & 48.39 & 27.97 & 54.16 & 37.73 & 42.06 & 0.50 & 45.00 & 42.22 & 56.25 & 42.86 & 0.00 & 37.27 & 0.40 \\
Kuwait & 59.29 & 27.97 & 41.30 & 15.89 & 36.11 & 0.40 & 14.81 & 33.33 & 25.00 & 45.24 & 0.00 & 23.68 & 0.30 \\
Lebanon & 55.39 & 29.32 & 50.98 & 58.00 & 48.42 & 0.50 & 37.59 & 32.04 & 37.50 & 42.86 & 0.00 & 30.00 & 0.30 \\
Libya* & 77.85 & 22.99 & 81.44 & 53.21 & 58.87 & 0.60 & 0.00 & 0.00 & 0.00 & 0.00 & 0.00 & 0.00 & 0.00 \\
Morocco & 55.83 & 18.58 & 56.08 & 22.18 & 38.17 & 0.40 & 6.48 & 24.63 & 18.75 & 52.38 & 0.00 & 20.45 & 0.30 \\
Oman & 57.65 & 21.03 & 62.88 & 12.60 & 38.54 & 0.40 & 36.11 & 29.26 & 12.50 & 47.62 & 0.00 & 25.10 & 0.30 \\
Qatar & 48.79 & 15.26 & 50.50 & 12.30 & 31.71 & 0.40 & 52.41 & 44.26 & 25.00 & 35.71 & 0.00 & 31.48 & 0.40 \\
Saudi Arabia & 64.94 & 30.92 & 37.20 & 28.84 & 40.48 & 0.50 & 28.70 & 34.44 & 25.00 & 38.10 & 0.00 & 25.25 & 0.30 \\
Syria* & 95.67 & 35.93 & 60.97 & 72.98 & 66.39 & 0.70 & 0.00 & 0.00 & 0.00 & 0.00 & 0.00 & 0.00 & 0.00 \\
Tunisia & 40.01 & 25.97 & 42.84 & 28.91 & 34.43 & 0.40 & 36.11 & 22.22 & 18.75 & 61.90 & 0.00 & 27.80 & 0.30 \\
UAE & 41.71 & 22.64 & 30.75 & 18.36 & 28.36 & 0.30 & 63.89 & 36.67 & 56.25 & 57.14 & 0.00 & 42.79 & 0.50 \\
Yemen* & 75.01 & 51.30 & 54.67 & 62.28 & 60.82 & 0.70 & 0.00 & 0.00 & 0.00 & 0.00 & 0.00 & 0.00 & 0.00 \\
\hline
\end{tabular}

Table 9

\begin{tabular}{|c|c|c|c|c|c|c|c|c|c|c|}
\hline$\alpha$ & 0.1 & 0.2 & 0.3 & 0.4 & 0.5 & 0.6 & 0.7 & 0.8 & 0.9 & 1 \\
\hline FSACC(MENA) & 0.8819 & 0.8498 & 0.8498 & 0.4339 & 0 & $\infty$ & $\infty$ & $\infty$ & $\infty$ & $\infty$ \\
\hline
\end{tabular}

Table 10 


\section{(iv) The Americas}

We shall use following abbreviations:

$D R=$ Dominican Republic

$T T=$ Trinidad and Tobago

\begin{tabular}{|c|c|c|c|c|c|c|c|c|c|c|c|c|c|}
\hline Name & $\epsilon_{1}$ & $\epsilon_{2}$ & $e_{3}$ & $e_{4}$ & Mean & FMV & $f_{1}$ & $f_{2}$ & $f_{3}$ & $f_{4}$ & $f_{5}$ & Mean & FMV \\
\hline Argentina & 35.63 & 18.50 & 34.04 & 5.36 & 28.38 & 0.30 & 49.26 & 59.81 & 87.50 & 78.57 & 0.00 & 55.03 & 0.60 \\
\hline Barbados & 9.70 & 15.41 & 7.00 & .00 & 30.78 & 0.40 & 39.44 & 29.26 & 50.00 & 38.10 & 0.00 & 31.36 & 0.40 \\
\hline Bolivia & 49.71 & 32.08 & 38.69 & 16.57 & 34.26 & 0.40 & 17.59 & 36.67 & 50.00 & 57.14 & 0.00 & 32.28 & 0.40 \\
\hline Brazil & 37.98 & 20.46 & 45.88 & 30.74 & 33.77 & 0.40 & 45.56 & 34.44 & 87.50 & 78.57 & 37.50 & 56.71 & 0.60 \\
\hline Canada & 17.59 & 23.64 & 15.48 & 36.11 & 23.21 & 0.30 & 57.41 & 68.52 & 62.50 & 64.29 & 0.00 & .54 & 0.60 \\
\hline Chy & 3 & 19.73 & 4.26 & 37 & .15 & 0.30 & 44.63 & 52.59 & 50.00 & 69.05 & 0.00 & 3.25 & .50 \\
\hline Colombia & 51.72 & 26.54 & 46.78 & 43.49 & 42.13 & 0.50 & 44.07 & 41.85 & 43.75 & 73.81 & 0.00 & 40.70 & 0.50 \\
\hline Costa & 36.35 & 24.40 & 3.82 & 23.97 & 29.63 & 0.30 & 56.48 & 45.56 & 37.50 & 71.43 & 0.00 & 42.19 & 0.50 \\
\hline Cuba & 53.60 & 26.60 & 34.00 & 13.99 & 32.05 & 0.40 & 25.00 & 17.78 & 12.50 & 38.10 & 0.00 & 18.68 & 0.20 \\
\hline DR & 47.97 & 33.84 & 46.38 & 24.33 & 38.13 & 0.40 & 49.26 & 59.07 & 62.50 & 64.29 & 0.00 & & 0.50 \\
\hline Ecua & 43.69 & 29.23 & 37.74 & 29.98 & 35.16 & 0.40 & 37.22 & 62.22 & 25.00 & 71.43 & 0.00 & 7 & 0.40 \\
\hline El Sal & 49.88 & 29.58 & 53.68 & 8.78 & 35.48 & 0.40 & 32.96 & 28.89 & 50.00 & 57.14 & $0 . c$ & 3.80 & 0.40 \\
\hline Guate & 48.75 & 34.25 & 57.12 & 20.33 & 40.11 & 0.50 & 37.59 & 51.48 & 56.25 & 64.29 & 0.00 & 11.92 & 0.50 \\
\hline Guyana & 39.35 & 25.57 & 83.50 & 5.81 & 38.56 & 0.40 & 25.74 & 50.74 & 25.00 & 52.38 & $0 . c$ & 30.77 & 0.40 \\
\hline Hai & 57.68 & 56.20 & 58.13 & 2.60 & 43.65 & 0.50 & 33.52 & 37.22 & 50.00 & 28.57 & 0.0 & 9.86 & 0.30 \\
\hline Honduras & 53.76 & 34.82 & 60.12 & 16.38 & 41.27 & 0.50 & 24.63 & 31.67 & 37.50 & 59.52 & 0.00 & 30.66 & 0.40 \\
\hline Jam & 43.21 & 30.91 & 46.13 & 8.68 & 23 & 0.40 & 47.78 & 61.85 & 81.25 & 54.76 & 0.00 & 13 & 0.50 \\
\hline & 43.03 & 30.36 & 84 & 61.85 & 47.02 & 0.50 & 45.00 & 63.15 & 50.00 & 73.81 & 0.00 & 39 & 0.50 \\
\hline $\mathrm{Nic}$ & 4 & 31.92 & 39.79 & 23.67 & 73 & 0.40 & 44.07 & 66.11 & 56.25 & 52.38 & 0. & 43.76 & 0.50 \\
\hline $\mathrm{Pa}$ & 46 & 29.14 & 46.18 & 23.55 & 34.08 & 0.40 & 11.85 & 73.15 & 37.50 & 57.14 & 0.00 & 35.93 & 0.40 \\
\hline Paras & 46.02 & 21.22 & 38.92 & 26.14 & 33.08 & 0.40 & 35.93 & 55.56 & 37.50 & 76.19 & 0.00 & 41.04 & 0.50 \\
\hline Peru & 43.85 & 31.98 & 44.81 & 30.33 & 37.74 & 0.40 & 47.78 & 28.33 & 62.50 & 61.90 & 0.00 & 40.10 & 0.50 \\
\hline Surinam & 36.66 & 12.35 & 67.00 & 38.67 & 38.67 & 0.40 & 20.56 & 19.44 & 31.25 & 40.48 & 0.00 & 22.35 & 0.30 \\
\hline TT & 35.88 & 14.40 & 67.00 & 16.26 & 33.38 & 0.40 & 31.11 & 44.63 & 31.25 & 66.67 & 0.00 & 4.73 & 0.40 \\
\hline United St & 20.42 & 23.51 & 20.96 & 45.10 & 27.50 & 0.30 & 96.30 & 79.63 & 68.75 & 78.57 & 75.00 & 79.65 & 0.80 \\
\hline & .05 & 20.69 & 28.36 & 13.89 & 22 & 0.30 & 4 & 43.70 & 43.75 & 78.57 & $0 . c$ & 41.32 & 0.50 \\
\hline Venezuela & 58.82 & 27.75 & 55.42 & 35.94 & 4.48 & 0.50 & 25.00 & 40.93 & 12.50 & 40.48 & 0.00 & 23.78 & 0.30 \\
\hline
\end{tabular}

Table 11 
662 S. Acharjee, D.J. Sarma, R.A. Hanneman, J.N. Mordeson and D.S. Malik

\begin{tabular}{|c|c|c|c|c|c|c|c|c|c|c|}
\hline$\alpha$ & 0.1 & 0.2 & 0.3 & 0.4 & 0.5 & 0.6 & 0.7 & 0.8 & 0.9 & 1 \\
\hline FSACC( The Americas) & 1 & 1 & 0.9813 & 0.7735 & 0.3162 & $\infty$ & $\infty$ & $\infty$ & $\infty$ & $\infty$ \\
\hline
\end{tabular}

Table 12

\section{(v) Europe}

We shall use following abbreviations:

$B H=$ Bosnia and Herzegovina 


\begin{tabular}{|c|c|c|c|c|c|c|c|c|c|c|c|c|c|}
\hline Name & $e_{1}$ & $e_{2}$ & $e_{3}$ & $e_{4}$ & Mean & FMV & $f_{1}$ & $f_{2}$ & $f_{3}$ & $f_{4}$ & $f_{5}$ & Mean & FMV \\
\hline Albania & 52.88 & 29.58 & 42.36 & 18.25 & 35.77 & 0.40 & 73.70 & 46.30 & 43.75 & 61.90 & 0.00 & 45.13 & 0.50 \\
\hline Austria & 21.47 & 18.14 & 14.05 & 33.14 & 21.70 & 0.30 & 57.96 & 59.07 & 68.75 & 76.19 & 0.00 & 52.39 & 0.60 \\
\hline Belgium & 5.42 & 19.83 & 24.03 & 31.28 & 25.14 & 0.30 & 71.30 & 50.74 & 75.00 & 71.43 & 0.00 & 53.69 & 0.60 \\
\hline $\mathrm{BH}$ & 58.88 & 25.21 & 32.99 & 26.43 & 35.88 & 0.40 & 57.41 & 43.70 & 31.25 & 69.05 & 0.00 & 40.28 & 0.50 \\
\hline Bulgaria & 40.41 & 20.54 & 33.79 & 30.50 & 31.31 & 0.40 & 43.15 & 38.52 & 43.75 & 71.43 & 0.00 & 39.37 & 0.40 \\
\hline Croatia & 36.51 & 25.29 & 36.88 & 16.54 & 28.80 & 0.30 & 69.63 & 70.19 & 56.25 & 76.19 & 0.00 & 54.45 & 0.60 \\
\hline Cyprus & 27.60 & 23.25 & 28.95 & 30.90 & 27.67 & 0.30 & 54.26 & 74.44 & 18.75 & 61.90 & 0.00 & 41.87 & 0.50 \\
\hline Czech Republic & 27.22 & 19.38 & 31.55 & 27.78 & 26.48 & 0.30 & 54.81 & 50.74 & 56.25 & 66.67 & 0.00 & 45.69 & 0.50 \\
\hline Denmark & 15.68 & 18.53 & 11.06 & 23.92 & 17.30 & 0.20 & 59.81 & 68.52 & 50.00 & 69.05 & 0.00 & 49.48 & 0.50 \\
\hline Estonia & 35.94 & 16.87 & 39.31 & 11.14 & 25.82 & 0.30 & 30.19 & 23.33 & 31.25 & 80.95 & 0.00 & 33.14 & 0.40 \\
\hline Finland & 22.07 & 19.69 & 19.08 & 22.66 & 20.88 & 0.30 & 52.78 & 62.04 & 56.25 & 66.67 & 0.00 & 47.55 & 0.50 \\
\hline France & 26.13 & 20.27 & 18.01 & 44.77 & 27.30 & 0.30 & 52.59 & 76.85 & 43.75 & 69.05 & 0.00 & 48.45 & 0.50 \\
\hline Germany & 23.61 & 19.97 & 20.81 & 40.76 & 26.29 & 0.30 & 61.67 & 64.81 & 43.75 & 78.57 & 0.00 & 49.76 & 0.50 \\
\hline Greec & 37.64 & 22.74 & 38.10 & 38.77 & 34.31 & 0.40 & 53.89 & 36.85 & 18.75 & 38.10 & 0.00 & 29.52 & 0.30 \\
\hline Hungary & 23.66 & 20.69 & 35.56 & 23.30 & 25.80 & 0.30 & 59.81 & 41.85 & 68.75 & 76.19 & 0.00 & 49.32 & 0.50 \\
\hline Iceland & 4.88 & 12.22 & 20.43 & 15.03 & 18.14 & 0.20 & 45.37 & 52.22 & 37.50 & 45.24 & 0.00 & 36.07 & 0.40 \\
\hline Ireland & 19.07 & 22.62 & 20.21 & 33.13 & 23.76 & 0.30 & 69.63 & 71.30 & 18.75 & 61.90 & 0.00 & 44.32 & 0.50 \\
\hline It. & 36.39 & 21.50 & 33.62 & 38.56 & 32.52 & 0.40 & 42.59 & 65.93 & 37.50 & 69.05 & 0.00 & 43.01 & 0.50 \\
\hline Kosovo & 55.11 & 39.25 & 42.13 & 15.94 & 38.11 & 0.40 & 48.15 & 59.92 & 43.75 & 47.62 & 0.00 & 39.89 & 0.40 \\
\hline Latvi: & 41.95 & 20.25 & 33.22 & 12.95 & 27.09 & 0.30 & 58.89 & 50.19 & 43.75 & 71.43 & 0.00 & 44.85 & 0.50 \\
\hline ithuani & 35.09 & 20.58 & 34.10 & 16.94 & 26.68 & 0.30 & 59.26 & 54.81 & 25.00 & 73.81 & 0.00 & 42.58 & 0.50 \\
\hline $\mathrm{Lu}$ & 22.99 & 18.64 & 9.76 & 49.43 & 25.20 & 0.30 & 33.15 & 31.67 & 68.75 & 26.19 & 0.00 & 31.95 & 0.40 \\
\hline Macedonia & 49.81 & 24.38 & 44.06 & 24.11 & 35.59 & 0.40 & 70.37 & 60.19 & 62.50 & 42.86 & 0.00 & 47.18 & 0.50 \\
\hline Montenegro & 41.68 & 22.15 & 38.08 & 23.45 & 31.34 & 0.40 & 69.63 & 60.56 & 56.25 & 59.52 & 0.00 & 49.19 & 0.50 \\
\hline $\mathrm{Ne}$ & 17.60 & 17.86 & 21 & 28.58 & 21.42 & 0.30 & 74.63 & 79.07 & 87.50 & 99.99 & 0.00 & 68.24 & 0.70 \\
\hline Norway & 17.88 & 19.90 & 14.85 & 34.90 & 21.88 & 0.30 & 65.93 & 82.41 & 37.50 & 69.05 & 0.00 & 50.98 & 0.60 \\
\hline Poland & 34.76 & 19.50 & 29.07 & 23.33 & 26.66 & 0.30 & 46.11 & 47.96 & 68.75 & 76.19 & 0.00 & 47.80 & 0.50 \\
\hline & 21.50 & 22.62 & 16.06 & 16.89 & 19.27 & 0.20 & 58.52 & 73.52 & 68.75 & 83.33 & 0.00 & 56.82 & 0.60 \\
\hline Ro & 39.26 & 25.86 & 31.83 & 18.74 & 28.92 & 0.30 & 37.59 & 36.85 & 56.25 & 50.00 & 0.00 & 36.14 & 0.40 \\
\hline Serbia & 47.80 & 21.75 & 30.24 & 27.05 & 31.71 & 0.40 & 61.67 & 76.67 & 31.25 & 54.76 & 0.00 & 44.87 & 0.50 \\
\hline Slovakia & 32.47 & 20.94 & 31.60 & 16.42 & 25.36 & 0.30 & 57.96 & 42.41 & 31.25 & 61.90 & 0.00 & 38.70 & 0.40 \\
\hline & 1.82 & 22.08 & & 13.72 & 21.53 & 0.30 & 52.04 & 51.30 & 56.25 & 76.19 & 0.00 & 47.16 & 0.50 \\
\hline Spain & 25.14 & 23.19 & 18.65 & 29.67 & 24.16 & 0.30 & 77.04 & 64.07 & 50.00 & 73.81 & 0.00 & 52.98 & 0.60 \\
\hline Sweden & 21.39 & 19.70 & 10.84 & 38.60 & 22.63 & 0.30 & 72.22 & 62.96 & 75.00 & 73.81 & 0.00 & 56.80 & 0.60 \\
\hline Switzerla & 16.93 & 16.60 & 13.69 & 30.98 & 19.55 & 0.20 & 60.19 & 64.81 & 25.00 & 73.81 & 0.00 & 44.76 & 0.50 \\
\hline Turl & 45.47 & 29.38 & 44.11 & 57.55 & 44.13 & 0.50 & 57.41 & 47.41 & 37.50 & 52.38 & 0.00 & 38.94 & 0.40 \\
\hline United Kingdom & 18.45 & 20.37 & 21.83 & 46.50 & 26.79 & 0.30 & 74.63 & 79.07 & 43.75 & 69.05 & 37.50 & 60.80 & 0.70 \\
\hline
\end{tabular}

Table 13

\begin{tabular}{|c|c|c|c|c|c|c|c|c|c|c|}
\hline$\alpha$ & 0.1 & 0.2 & 0.3 & 0.4 & 0.5 & 0.6 & 0.7 & 0.8 & 0.9 & 1 \\
\hline FSACC( Europe) & 1 & 1 & 0.9444 & 0.4743 & 0 & $\infty$ & $\infty$ & $\infty$ & $\infty$ & $\infty$ \\
\hline
\end{tabular}

Table 14 
664 S. Acharjee, D.J. Sarma, R.A. Hanneman, J.N. Mordeson and D.S. Malik

\section{(vi) Sub-Saharan Africa}

We shall use following abbreviations:

$C A R=$ Central African Republic

$D R C=$ Democratic Republic of the Congo

$R C=$ Republic of the Congo 


\begin{tabular}{|c|c|c|c|c|c|c|c|c|c|c|c|c|c|}
\hline Name & $\sigma_{1}$ & $e_{2}$ & $e_{3}$ & 64 & Mean & FMV & $f_{1}$ & $f_{2}$ & $f_{3}$ & $f_{4}$ & $f_{0}$ & Mean & FMV \\
\hline Angola & 56.92 & 49.54 & 45.95 & $25 \% 2$ & 44.21 & 0.50 & 20.37 & 20.37 & 31.25 & 28.57 & 0.00 & 20.11 & 0.90 \\
\hline Benin & 46.95 & 36.52 & 39.70 & 14.90 & 94.52 & 0.40 & 98.70 & 20.56 & 56.25 & 66.67 & 0.00 & 36.44 & 0.40 \\
\hline Botswana & 97.25 & 42.82 & 46.38 & 19.21 & 96.41 & 0.40 & 24.81 & 1481 & 37.50 & 42.86 & 0.00 & 24.00 & 0.90 \\
\hline Burkina Faso & 59.55 & 40.77 & 40.73 & 25.96 & 41.76 & 0.50 & 47.41 & 90.56 & 37.50 & 42.86 & 0.00 & 31.67 & 0.40 \\
\hline Burundi & 6408 & 52.22 & 51.40 & 37.17 & 51.22 & 0.60 & 29.63 & 1481 & 18.75 & 38.10 & 0.00 & 20.26 & 0.90 \\
\hline Cameroon & 61.91 & 49.37 & 47.95 & 52.51 & 51.28 & 0.60 & 30.97 & 39.89 & 37.50 & 47.62 & 0.00 & 29.88 & 0.90 \\
\hline Cape Verde & 99.99 & 20.90 & 55.40 & 96.34 & 96.34 & 0.40 & 15.19 & 15.37 & 25.00 & 90.95 & 0.00 & 17.90 & 0.20 \\
\hline CAR & 89.67 & 48.67 & 85.43 & 62.21 & 70,00 & 0.70 & 14.81 & 17.22 & 12.50 & 7.14 & 0.00 & 10.39 & 0.20 \\
\hline Chad & 70.47 & 49.96 & 47.98 & 40.94 & 52.94 & 0.60 & 26.85 & 1204 & 31.25 & 52.38 & 0.00 & 24.50 & 0.90 \\
\hline $\mathrm{DRC}$ & 78.42 & 56.39 & 56.72 & 82.49 & 68.47 & 0.70 & 7.78 & 11.67 & 31.25 & 26.19 & 0.00 & 15.38 & 0.20 \\
\hline Djibouti & 49.19 & 49.61 & 55.25 & 92.42 & 45.10 & 0.50 & 25.00 & 28.89 & 37.50 & 59.52 & 0.00 & 30.18 & 0.40 \\
\hline Equatorial Guines & 56.66 & 40.62 & 46.98 & 1.00 & 96.16 & 0.40 & 0.00 & 18.52 & 0.00 & 23.81 & 0.00 & 8.47 & 0.10 \\
\hline Eritrea & 59.44 & 51.05 & 62.88 & 24.82 & 49.55 & 0.50 & 0.00 & 2.96 & 0,00 & 26.19 & 0.00 & 5,89 & 0.10 \\
\hline Ethiopia & 59.75 & 54.68 & 34.16 & 59.77 & 52.09 & 0.60 & 21.30 & 39.99 & 62.50 & 52.38 & 0.00 & 33.90 & 0.40 \\
\hline Gabon & 51.58 & 31.51 & 42.98 & 16.97 & 95.61 & 0.40 & 30.00 & 24.26 & 25.00 & 45.24 & 0.00 & 24.90 & 0.90 \\
\hline Gambis & 59.90 & 29.27 & 74.18 & 22.69 & 46.34 & 0.50 & 22.59 & 35.19 & 37.50 & 45.24 & 0.00 & 28.10 & 0.90 \\
\hline Ghana & 5189 & 38.42 & 47.45 & 28.26 & 41.50 & 0.50 & 22.04 & 90.19 & 25.00 & 45.24 & 0.00 & 24.49 & 0.90 \\
\hline Guines & 66,89 & 41.58 & 52.68 & 28.67 & 47.45 & 0.50 & 2.78 & 12.96 & 31.25 & 28.57 & 0.00 & 15.11 & 0.20 \\
\hline Guines-Bissau & 62.08 & 40.50 & 70.25 & 22.47 & 48,82 & 0.50 & 14.81 & 9204 & 25.00 & 21.49 & 0.00 & 18.66 & 0.20 \\
\hline Ivory & 62.07 & 38.72 & 46.22 & 39.45 & 45.11 & 0.50 & 37.96 & 90.19 & 50.00 & 30.95 & 0.00 & 29.82 & 0.90 \\
\hline Kenys & 54.53 & 52.84 & 46.75 & 72.28 & 56.60 & 0.60 & 21.85 & 27.41 & 6.25 & 42.86 & 0.00 & 19.67 & 0.20 \\
\hline Lesotho & 40.99 & 52.26 & 68.23 & 9.58 & 42.60 & 0.50 & 91.48 & 25.74 & 50.00 & 59.52 & 0.00 & 38.35 & 0.40 \\
\hline Liberia & 57.99 & 44.62 & 44.45 & 29.43 & 44.11 & 0.50 & 27.22 & 27.41 & 31.25 & 50.00 & 0.00 & 27.18 & 0.90 \\
\hline Madagasear & 50.97 & 50.87 & 52.86 & 15.99 & 42.52 & 0.50 & 31.30 & 14.44 & 31.25 & 42.86 & 0.00 & 23.97 & 0.90 \\
\hline Malawi & 54.69 & 56.74 & 47.78 & 2106 & 45,05 & 0.50 & 32.22 & 21.11 & 12.50 & 38.10 & 0.00 & 20.79 & 0.90 \\
\hline Mali & 64.04 & 34.08 & 31.69 & 57.41 & 46.80 & 0.50 & 15.19 & 12.04 & 43.75 & 93.93 & 0.00 & 20.86 & 0.90 \\
\hline Mauritania & 65.96 & 40.54 & 49.85 & 90.74 & 46.77 & 0.50 & 25.00 & $\$ 2.41$ & 12.50 & 40.48 & 0.00 & 22.08 & 0.90 \\
\hline Mauritius & 29.24 & 24.49 & 26.58 & 1.00 & 20.39 & 0.30 & 34.44 & 27.41 & 18.75 & 45.24 & 0.00 & 25.17 & 0.90 \\
\hline Mozambique & 39.91 & 48.46 & 54.40 & 35.96 & 44.66 & 0.50 & 53.89 & 50.56 & 12.50 & 47.62 & 0.00 & 32.91 & 0.40 \\
\hline Namibia & 99,00 & 49.68 & 51.42 & 18.27 & 98,09 & 0.40 & 28.15 & 2204 & 31.25 & 52.38 & 0.00 & 26.76 & 0.90 \\
\hline Niger & 57.70 & 48.17 & 42.13 & 40.98 & 47,09 & 0.50 & 12.41 & 29.26 & 25.00 & 40.48 & 0.00 & 21.43 & 0.90 \\
\hline Nigeria & 60.94 & 47.84 & 59.76 & 90.84 & 62.34 & 0.70 & 50.74 & 59.69 & 50.00 & 45.24 & 0.00 & 41.12 & 0.50 \\
\hline $\mathrm{RC}$ & 65.17 & 44.69 & 52.43 & 28.94 & 47.81 & 0.50 & 22.22 & 8.89 & 37.50 & 35.71 & 0.00 & 20.86 & 0.90 \\
\hline Rwanda & 55.44 & 47.65 & 46.23 & 42,00 & 47,89 & 0.50 & 30.93 & 45.19 & 31.25 & 54.76 & 0.00 & 32.49 & 0.40 \\
\hline Senegal & 44.97 & 42.31 & 36.96 & 35.48 & 99.99 & 0.40 & 49.68 & $\$ 2.59$ & 25.00 & 54.76 & 0.00 & 32.40 & 0.40 \\
\hline Sierra Leone & 50.57 & 53.29 & 41.72 & 17.70 & 40.82 & 0.50 & 44.44 & 45.56 & 43.75 & 54.76 & 0.00 & 37.70 & 0.40 \\
\hline Somalia & 7309 & 64.82 & 55.97 & 74.46 & 67.07 & 0.70 & 0.00 & 0.00 & 0.00 & 0.00 & 0.00 & 0,00 & 0.00 \\
\hline South Africs & 40.27 & 49.06 & 58.30 & 4184 & 45.87 & 0.50 & 38.89 & 55.74 & 31.25 & 64.29 & 0.00 & 38.03 & 0.40 \\
\hline South Sudsn & 74.79 & 50.70 & 60.80 & 76.15 & 65.59 & 0.70 & 20.37 & 1.48 & 18.75 & 28.57 & 0.00 & 13.89 & 0.20 \\
\hline & 80.64 & 54.12 & 46.18 & 85.04 & 66.50 & 0.70 & 24.07 & 27.41 & 6.25 & 33.93 & 0.00 & 18.21 & 0.20 \\
\hline Swaziland & 57.69 & 53.88 & 67.33 & 15.39 & 48.57 & 0.50 & 36.30 & 22.04 & 37.50 & 42.86 & 0.00 & 27.74 & 0.90 \\
\hline Tanzania & 51.66 & 54.67 & 47.66 & 40.46 & 48.61 & 0.50 & 27.04 & 26.48 & 25.00 & 47.62 & 0.00 & 25.29 & 0.90 \\
\hline Togo & 64.78 & 39.82 & 47.78 & 24.09 & 44.12 & 0.50 & 26.48 & 4.68 & 31.25 & 19.05 & 0.00 & 16.28 & 0.20 \\
\hline Uganda & 54.89 & 52.18 & 39.45 & 48.73 & 48.81 & 0.50 & 50.93 & 50.19 & 37.50 & 59.52 & 0.00 & 39.69 & 0.40 \\
\hline Zambia & 45.10 & 58.76 & 50.03 & 24.46 & 44.59 & 0.50 & 93.89 & 29.81 & 43.75 & 98.10 & 0.00 & 29.11 & 0.90 \\
\hline Zimbabwe & 60.28 & 52.25 & 48.92 & 26.78 & 4706 & 0.50 & 15.37 & 20.56 & 12.50 & 42.86 & 0.00 & 18.26 & 0.20 \\
\hline
\end{tabular}

Table 15 
666 S. Acharjee, D.J. Sarma, R.A. Hanneman, J.N. Mordeson and D.S. Malik

\begin{tabular}{|c|c|c|c|c|c|c|c|c|c|c|}
\hline$\alpha$ & 0.1 & 0.2 & 0.3 & 0.4 & 0.5 & 0.6 & 0.7 & 0.8 & 0.9 & 1 \\
\hline FSACC(Sub-Saharan Africa) & 0.9891 & 0.9668 & 0.8470 & 0.5164 & 0.1622 & $\infty$ & $\infty$ & $\infty$ & $\infty$ & $\infty$ \\
\hline
\end{tabular}

Table 16

\section{Analysis}

\begin{tabular}{|c|c|c|c|c|c|c|c|c|c|c|}
\hline$\alpha$ & 0.1 & 0.2 & 0.9 & 0.4 & 0.5 & 0.6 & 0.7 & 0.8 & 0.9 & 1 \\
\hline FSACC(Asia Pacific) & 0.9623 & 0.9428 & 0.8292 & 0.6191 & 0.9273 & 0 & $\infty$ & $\infty$ & $\infty$ & $\infty$ \\
\hline FSAOC(Russia and Eurasia) & 1 & 1 & 1 & 0.8165 & 0 & $\infty$ & $\infty$ & $\infty$ & $\infty$ & \\
\hline FSACC(MENA) & 0.8819 & 0.8496 & 0.8498 & 0.4399 & 0 & $\infty$ & $\infty$ & $\infty$ & $\infty$ & \\
\hline FSACC(The Americas) & 1 & 1 & 0.9813 & 0.7795 & 0.3162 & $\infty$ & $\infty$ & $\infty$ & $\infty$ & \\
\hline FSACC(Europe) & 1 & 1 & 0.9444 & 0.4743 & 0 & $\infty$ & $\infty$ & $\infty$ & $\infty$ & $\infty$ \\
\hline FSACO(Sub-Sahsan Africa) & 0.9891 & 0.9668 & 0.8470 & 0.5164 & 0.1622 & $\infty$ & $\infty$ & $\infty$ & $\infty$ & $\infty$ \\
\hline
\end{tabular}

Table 17

Thus, The Americas and Europe have the highest correlation levels in this case.

\section{Complement of vulnerability and government responses}

\section{(i) Asia Pacific}

Complement of vulnerability and government responses of the region "Asia Pacific" is shown in Table 18. 


\begin{tabular}{|c|c|c|c|c|c|}
\hline Name & Mean of vulnerability & 100 - Mean of vulnerability & FMV & Mean of govt. responses & FMV \\
\hline Afghanistan* & 67.06 & 32.94 & 0.40 & 0.00 & 0.00 \\
\hline Australia & 21.98 & 78.02 & 0.80 & 59.32 & 0.60 \\
\hline Bangladesh & 44.12 & 55.88 & 0.60 & 45.62 & 0.50 \\
\hline Brunei & 63.92 & 36.08 & 0.40 & 17.32 & 0.20 \\
\hline Cambodia & 41.51 & 58.49 & 0.60 & 32.06 & 0.40 \\
\hline China & 44.66 & 55.34 & 0.60 & 28.58 & 0.30 \\
\hline HSC & 29.23 & 70.77 & 0.80 & 8.41 & 0.10 \\
\hline India & 51.35 & 48.65 & 0.50 & 35.61 & 0.40 \\
\hline Indonesia & 42.22 & 57.78 & 0.60 & 36.58 & 0.40 \\
\hline Japan & 21.40 & 78.60 & 0.80 & 25.20 & 0.30 \\
\hline Laos & 36.45 & 63.55 & 0.70 & 27.29 & 0.30 \\
\hline Malaysia & 38.51 & 61.49 & 0.70 & 30.98 & 0.40 \\
\hline Mongolia & 30.09 & 69.91 & 0.70 & 27.66 & 0.30 \\
\hline Myanmar & 56.36 & 43.64 & 0.50 & 30.00 & 0.30 \\
\hline Nepal & 40.37 & 59.63 & 0.60 & 43.57 & 0.50 \\
\hline New Zealand & 18.29 & 81.71 & 0.90 & 46.70 & 0.50 \\
\hline North Korea & 45.84 & 54.16 & 0.60 & -4.21 & 0.00 \\
\hline Pakistan & 62.47 & 37.53 & 0.40 & 33.35 & 0.40 \\
\hline $\mathrm{PNG}$ & 59.01 & 40.99 & 0.50 & 13.89 & 0.20 \\
\hline Philippines & 47.67 & 52.33 & 0.60 & 47.57 & 0.50 \\
\hline Singapore & 18.38 & 81.62 & 0.90 & 20.28 & 0.30 \\
\hline South Korea & 29.79 & 70.22 & 0.80 & 22.72 & 0.30 \\
\hline Sri Lanka & 36.26 & 63.74 & 0.70 & 37.06 & 0.40 \\
\hline Taiwan & 23.10 & 76.90 & 0.80 & 32.16 & 0.40 \\
\hline Thailand & 47.54 & 52.46 & 0.60 & 37.85 & 0.40 \\
\hline Timor-Leste & 39.13 & 60.88 & 0.70 & 18.47 & 0.20 \\
\hline Vietnam & 29.34 & 70.66 & 0.80 & 41.69 & 0.50 \\
\hline
\end{tabular}

Table 18

\begin{tabular}{|c|c|c|c|c|c|c|c|c|c|c|}
\hline$\alpha$ & 0.1 & 0.2 & 0.3 & 0.4 & 0.5 & 0.6 & 0.7 & 0.8 & 0.9 & 1 \\
\hline FSACC $^{C}$ (Asia Pacific) & 0.9623 & 0.9428 & 0.8819 & 0.7201 & 0.5000 & 0.2182 & $\infty$ & $\infty$ & $\infty$ & $\infty$ \\
\hline
\end{tabular}

Table 19 
668 S. Acharjee, D.J. Sarma, R.A. Hanneman, J.N. Mordeson and D.S. Malik

\begin{tabular}{|c|c|c|c|c|c|}
\hline Name & Mean of vulnerability & 100- Mean of vulnerability & FMV & Mean of govt. responses & FMV \\
\hline height Armenia & 37.14 & 62.86 & 0.70 & 40.88 & 0.50 \\
Azerbaijan & 37.09 & 62.91 & 0.70 & 32.92 & 0.40 \\
Belarus & 34.64 & 65.36 & 0.70 & 31.52 & 0.40 \\
Georgia & 34.95 & 65.05 & 0.70 & 48.41 & 0.50 \\
Kazakhstan & 32.86 & 67.14 & 0.70 & 27.37 & 0.30 \\
Kyrgyzstan & 35.18 & 64.81 & 0.70 & 30.24 & 0.40 \\
Moldova & 32.04 & 67.96 & 0.70 & 45.49 & 0.50 \\
Russia & 43.45 & 56.55 & 0.60 & 24.84 & 0.30 \\
Tajikistan & 42.42 & 57.58 & 0.60 & 31.24 & 0.40 \\
Turkmenistan & 37.34 & 62.66 & 0.70 & 23.56 & 0.30 \\
Ukraine & 40.64 & 59.36 & 0.60 & 36.84 & 0.40 \\
Uzbekistan & 36.80 & 63.20 & 0.70 & 23.82 & 0.30 \\
\hline
\end{tabular}

Table 20

\begin{tabular}{|c|c|c|c|c|c|c|c|c|c|c|}
\hline$\alpha$ & 0.1 & 0.2 & 0.3 & 0.4 & 0.5 & 0.6 & 0.7 & 0.8 & 0.9 & 1 \\
\hline FSACC $^{C}$ (Russia and Eurasia) & 1 & 1 & 1 & 0.8165 & 0.5000 & $\infty$ & $\infty$ & $\infty$ & $\infty$ & $\infty$ \\
\hline
\end{tabular}

Table 21 


\section{(iii) The Middle East and North Africa}

\begin{tabular}{|c|c|c|c|c|c|}
\hline Name & Mean of vulnerability & 100 - Mean of vulnerability & FMV & Mean of govt. responses & FMV \\
\hline Algeria & 44.40 & 55.60 & 0.60 & 24.09 & 0.30 \\
Bahrain & 40.06 & 59.94 & 0.60 & 26.81 & 0.30 \\
Egypt & 49.24 & 50.76 & 0.60 & 34.03 & 0.40 \\
Iran & 50.93 & 49.07 & 0.50 & 4.82 & 0.10 \\
Iraq* & 63.82 & 36.18 & 0.40 & 0.00 & 0.00 \\
Israel & 36.87 & 63.13 & 0.70 & 41.93 & 0.50 \\
Jordan & 42.06 & 57.94 & 0.60 & 37.27 & 0.40 \\
Kuwait & 36.11 & 63.89 & 0.70 & 23.68 & 0.30 \\
Lebanon & 48.42 & 51.58 & 0.60 & 30.00 & 0.30 \\
Libya* & 58.87 & 41.13 & 0.50 & 0.00 & 0.00 \\
Morocco & 38.17 & 61.83 & 0.70 & 20.45 & 0.30 \\
Oman & 38.54 & 61.46 & 0.70 & 25.10 & 0.30 \\
Qatar & 31.71 & 68.29 & 0.70 & 31.48 & 0.40 \\
Saudi Arabia & 40.48 & 59.52 & 0.60 & 25.25 & 0.30 \\
Syria* & 66.39 & 33.61 & 0.40 & 0.00 & 0.00 \\
Tunisia & 34.43 & 65.57 & 0.70 & 27.80 & 0.30 \\
UAE & 28.36 & 71.64 & 0.80 & 42.79 & 0.50 \\
Yemen* & 60.82 & 39.18 & 0.40 & 0.00 & 0.00 \\
\hline
\end{tabular}

Table 22

\begin{tabular}{|c|c|c|c|c|c|c|c|c|c|c|}
\hline$\alpha$ & 0.1 & 0.2 & 0.3 & 0.4 & 0.5 & 0.6 & 0.7 & 0.8 & 0.9 & 1 \\
\hline FSACC $^{C}(\mathrm{MENA})$ & 0.8819 & 0.8498 & 0.8498 & 0.5270 & 0.3651 & $\infty$ & $\infty$ & $\infty$ & $\infty$ & $\infty$ \\
\hline
\end{tabular}

Table 23

(iv) The Americas

We shall use following abbreviations:

$D R=$ Dominican Republic

$T T=$ Trinidad and Tobago 
670 S. Acharjee, D.J. Sarma, R.A. Hanneman, J.N. Mordeson and D.S. Malik

\begin{tabular}{|c|c|c|c|c|c|}
\hline Name & Mean of vulnerability & 100 - Mean of vulnerability & FMV & Mean of govt. reaponses & FMV \\
height Argentina & 28.38 & 71.62 & 0.80 & 55.03 & 0.60 \\
Barbados & 30.78 & 69.22 & 0.70 & 31.36 & 0.40 \\
Bolivia & 34.26 & 65.74 & 0.70 & 32.28 & 0.40 \\
Brazil & 33.77 & 66.23 & 0.70 & 56.71 & 0.60 \\
Canada & 23.21 & 76.80 & 0.80 & 50.54 & 0.60 \\
Chile & 29.15 & 70.85 & 0.80 & 43.25 & 0.50 \\
Colombia & 42.13 & 57.87 & 0.60 & 40.70 & 0.50 \\
Costa Rica & 29.63 & 70.37 & 0.80 & 42.19 & 0.50 \\
Cuba & 32.05 & 67.95 & 0.70 & 18.68 & 0.20 \\
DR & 38.13 & 61.87 & 0.70 & 47.02 & 0.50 \\
Ecuador & 35.16 & 64.84 & 0.70 & 39.17 & 0.40 \\
El Salvador & 35.48 & 64.52 & 0.70 & 33.80 & 0.40 \\
Guatemala & 40.11 & 59.89 & 0.60 & 41.92 & 0.50 \\
Guyana & 38.56 & 61.44 & 0.70 & 30.77 & 0.40 \\
Haiti & 43.65 & 56.35 & 0.60 & 29.86 & 0.30 \\
Honduras & 41.27 & 58.73 & 0.60 & 30.66 & 0.40 \\
Jamaica & 32.23 & 67.77 & 0.70 & 49.13 & 0.50 \\
Mexico & 47.02 & 52.98 & 0.60 & 46.39 & 0.50 \\
Nicaragua & 34.73 & 65.27 & 0.70 & 43.76 & 0.50 \\
Panama & 34.08 & 65.92 & 0.70 & 35.93 & 0.40 \\
Paraguay & 33.08 & 66.92 & 0.70 & 41.04 & 0.50 \\
Peru & 37.74 & 62.26 & 0.70 & 40.10 & 0.50 \\
Suriname & 38.67 & 61.33 & 0.70 & 22.35 & 0.30 \\
TT & 33.38 & 66.62 & 0.70 & 34.73 & 0.40 \\
United States & 27.50 & 72.50 & 0.80 & 79.65 & 0.80 \\
Uruguay & 22.40 & 77.60 & 0.80 & 41.32 & 0.50 \\
Venezuela & 44.48 & 55.52 & 0.60 & 23.78 & 0.30 \\
\hline
\end{tabular}

Table 24 


\section{(v) Europe}

We shall use following abbreviations:

$B H=$ Bosnia and Herzegovina 
672 S. Acharjee, D.J. Sarma, R.A. Hanneman, J.N. Mordeson and D.S. Malik

\begin{tabular}{|c|c|c|c|c|c|}
\hline Name & Mean of vulnerability & 100 - Mean of vulnerability & FMV & Mean of govt. responses & FMV \\
\hline Albania & 35.77 & 64.23 & 0.70 & 45.13 & 0.50 \\
\hline Austria & 21.70 & 78.30 & 0.80 & 52.39 & 0.60 \\
\hline Belgium & 25.14 & 74.86 & 0.80 & 53.69 & 0.60 \\
\hline $\mathrm{BH}$ & 35.88 & 64.12 & 0.70 & 40.28 & 0.50 \\
\hline Bulgaria & 31.31 & 68.69 & 0.70 & 39.37 & 0.40 \\
\hline Croatia & 28.80 & 71.19 & 0.80 & 54.45 & 0.60 \\
\hline Cyprus & 27.67 & 72.33 & 0.80 & 41.87 & 0.50 \\
\hline Czech Republic & 26.48 & 73.52 & 0.80 & 45.69 & 0.50 \\
\hline Denmark & 17.30 & 82.70 & 0.90 & 49.48 & 0.50 \\
\hline Estonia & 25.82 & 74.19 & 0.80 & 33.14 & 0.40 \\
\hline Finland & 20.88 & 79.13 & 0.80 & 47.55 & 0.50 \\
\hline France & 27.30 & 72.70 & 0.80 & 48.45 & 0.50 \\
\hline Germany & 26.29 & 73.71 & 0.80 & 49.76 & 0.50 \\
\hline Greece & 34.31 & 65.69 & 0.70 & 29.52 & 0.30 \\
\hline Hungary & 25.80 & 74.20 & 0.80 & 49.32 & 0.50 \\
\hline Iceland & 18.14 & 81.86 & 0.90 & 36.07 & 0.40 \\
\hline Ireland & 23.76 & 76.24 & 0.80 & 44.32 & 0.50 \\
\hline Italy & 32.52 & 67.48 & 0.70 & 43.01 & 0.50 \\
\hline Kosovo & 28.11 & 61.89 & 0.70 & 39.89 & 0.40 \\
\hline Latvia & 27.09 & 72.91 & 0.80 & 44.85 & 0.50 \\
\hline Lithuania & 26.68 & 73.32 & 0.80 & 42.58 & 0.50 \\
\hline Luxembourg & 25.20 & 74.80 & 0.80 & 31.95 & 0.40 \\
\hline Macedonia & 35.59 & 64.41 & 0.70 & 47.18 & 0.50 \\
\hline Montenegro & 31.34 & 68.66 & 0.70 & 49.19 & 0.50 \\
\hline Netherlands & 21.42 & 78.58 & 0.80 & 68.24 & 0.70 \\
\hline Norway & 21.88 & 78.12 & 0.80 & 50.98 & 0.60 \\
\hline Poland & 26.66 & 73.34 & 0.80 & 47.80 & 0.50 \\
\hline Portugal & 19.27 & 80.73 & 0.90 & 56.82 & 0.60 \\
\hline Romania & 28.92 & 71.08 & 0.80 & 36.14 & 0.40 \\
\hline Serbia & 31.71 & 68.29 & 0.70 & 44.87 & 0.50 \\
\hline Slovakia & 25.36 & 74.64 & 0.80 & 38.70 & 0.40 \\
\hline Slovenia & 21.53 & 78.47 & 0.80 & 47.16 & 0.50 \\
\hline Spain & 24.16 & 75.84 & 0.80 & 52.98 & 0.60 \\
\hline Sweden & 22.63 & 77.37 & 0.80 & 56.80 & 0.60 \\
\hline Switzerland & 19.55 & 80.45 & 0.90 & 44.76 & 0.50 \\
\hline Turkey & 44.13 & 55.87 & 0.60 & 38.94 & 0.40 \\
\hline United Kingdom & 26.79 & 73.21 & 0.80 & 60.80 & 0.70 \\
\hline
\end{tabular}

Table 26 


\begin{tabular}{|c|c|c|c|c|c|c|c|c|c|c|}
\hline$\alpha$ & 0.1 & 0.2 & 0.3 & 0.4 & 0.5 & 0.6 & 0.7 & 0.8 & 0.9 & 1 \\
\hline FSACC $^{C}$ (Europe) & 1 & 1 & 1 & 0.9864 & 0.8699 & 0.4932 & 0.2357 & $\infty$ & $\infty$ & $\infty$ \\
\hline
\end{tabular}

Table 27

(vi) Sub-Saharan Africa

We shall use following abbreviations:

$C A R=$ Central African Republic

$D R C=$ Democratic Republic of the Congo

$R C=$ Republic of the Congo 
674 S. Acharjee, D.J. Sarma, R.A. Hanneman, J.N. Mordeson and D.S. Malik

\begin{tabular}{|c|c|c|c|c|c|}
\hline Name & Mean of vulnerability & 100 - Menn of vulnerability & FMV & Mean of govt. responses & FMV \\
\hline Angoln & 44.21 & 55.79 & 0.60 & 20.11 & 0.90 \\
\hline Benin & $\$ 4.52$ & 65.48 & 0.70 & 38.44 & 0.40 \\
\hline Botswann & 36.41 & 63.59 & 0.70 & 24.00 & 0.30 \\
\hline Burkina Faso & 41.76 & 58.24 & 0.60 & 31.67 & 0.40 \\
\hline Burundi & 51.22 & 48.78 & 0.50 & 20.26 & 0.30 \\
\hline Cameroon & 51.28 & 48.72 & 0.50 & 29.88 & 0.30 \\
\hline Cape Verde & S6.34 & 63.86 & 0.70 & 17.30 & 0.20 \\
\hline CAR & 7000 & 30.00 & 0.40 & 10.33 & 0.20 \\
\hline Chad & 52.34 & 47.66 & 0.50 & 24.50 & 0.30 \\
\hline DRC & 68.47 & 31.53 & 0.40 & 15.38 & 0.20 \\
\hline Djibouti & 45.10 & 54.90 & 0.80 & 30.18 & 0.40 \\
\hline Equatorial Guinea & 38.16 & 63.84 & 0.70 & 8.47 & 0.10 \\
\hline Eritrea & 49.55 & 50.45 & 0.60 & 5.83 & 0.10 \\
\hline Ethiopis & 5209 & 47.91 & 0.50 & 39.90 & 0.40 \\
\hline Gabon & 35.61 & 64.39 & 0.70 & 24.90 & 0.30 \\
\hline Gambia & 46.34 & 53.66 & 0.60 & 23.10 & 0.30 \\
\hline Ghana & 41.50 & 58.50 & 0.60 & 24.49 & 0.30 \\
\hline Guinea & 47.45 & 52.55 & 0.60 & 15.11 & 0.20 \\
\hline Guinea-Bissau & 48.82 & 51.18 & 0.60 & 18.66 & 0.20 \\
\hline Ivary Const & 45.11 & 54.89 & 0.80 & 29.82 & 0.30 \\
\hline Kenya & 56.60 & 43.40 & 0.50 & 19.67 & 0.20 \\
\hline Lesotho & 42.60 & 57.40 & 0.60 & 39.35 & 0.40 \\
\hline Liberin & 44.11 & 55.89 & 0.80 & 27.18 & 0.30 \\
\hline Madagaxar & 42.52 & 57.48 & 0.60 & 23.97 & 0.30 \\
\hline Malnwi & 4505 & 54.95 & 0.60 & 20.79 & 0.30 \\
\hline Mali & 4680 & 53.20 & 0.60 & 20.86 & 0.30 \\
\hline Mauritania & 46.77 & 53.25 & 0.60 & 22.08 & 0.30 \\
\hline Mauritius & 20.33 & 79.67 & 0.80 & 25.17 & 0.30 \\
\hline Mozambique & 44.66 & 55.34 & 0.20 & 32.91 & 0.40 \\
\hline Namibis & 3809 & 61.91 & 0.70 & 28.76 & 0.30 \\
\hline Niger & 4709 & 52.91 & 0.60 & 21.43 & 0.30 \\
\hline Nigeria & 62.34 & 37.86 & 0.40 & $41: 12$ & 0.50 \\
\hline $\mathrm{RC}$ & 4781 & 52.19 & 0.60 & 20.86 & 0.30 \\
\hline Rwanda & 4783 & 52.17 & 0.60 & 32.43 & 0.40 \\
\hline Senegal & 39.93 & 60.07 & 0.70 & 32.40 & 0.40 \\
\hline Sierra Loone & 4082 & 59.18 & 0.60 & 37.70 & 0.40 \\
\hline Somalia & 6707 & 32.93 & 0.40 & 0.00 & 0.00 \\
\hline South Africa & 4587 & 54.13 & 0.60 & 38.03 & 0.40 \\
\hline South Sudan & 65.59 & 34.41 & 0.40 & 13.83 & 0.20 \\
\hline Sudan & 66.50 & 33.50 & 0.40 & 18.21 & 0.20 \\
\hline Swaziland & 48.57 & 51.43 & 0.60 & 27.74 & 0.30 \\
\hline Tanzania & 48.61 & 51.39 & 0.60 & 25.23 & 0.30 \\
\hline Togo & 44.12 & 55.88 & 0.60 & 16.28 & 0.20 \\
\hline Uganda & 48.81 & 51.19 & 0.60 & 39.63 & 0.40 \\
\hline Zambia & 44.59 & 55.41 & 0.60 & 29.11 & 0.30 \\
\hline Zimbabme & 4706 & 52.94 & 0.80 & 18.26 & 0.20 \\
\hline
\end{tabular}

Table 28 


\begin{tabular}{|c|c|c|c|c|c|c|c|c|c|c|}
\hline$\alpha$ & 0.1 & 0.2 & 0.3 & 0.4 & 0.5 & 0.6 & 0.7 & 0.8 & 0.9 & 1 \\
\hline FSACC $^{C}$ (Sub-Saharan Africa) & 0.9891 & 0.9668 & 0.8470 & 0.5108 & 0 & $\infty$ & $\infty$ & $\infty$ & $\infty$ & $\infty$ \\
\hline
\end{tabular}

Table 29

\section{Analysis}

MENA $=$ Middle East and North Africa

\begin{tabular}{|c|c|c|c|c|c|c|c|c|c|c|}
\hline$\alpha$ & 0.1 & 0.2 & 0.3 & 0.4 & 0.5 & 0.6 & 0.7 & 0.8 & 0.9 & 1 \\
\hline FSACC $^{C}$ (Asia Pacific) & 0.9623 & 0.9428 & 0.8819 & 0.7201 & 0.5000 & 0.2182 & $\infty$ & $\infty$ & $\infty$ & $\infty$ \\
FSACC $^{C}$ (Russia and Eurasia) & 1 & 1 & 1 & 0.8165 & 0.5000 & $\infty$ & $\infty$ & $\infty$ & $\infty$ & $\infty$ \\
FSACC $^{C}$ (MENA) & 0.8819 & 0.8498 & 0.8498 & 0.5270 & 0.3651 & $\infty$ & $\infty$ & $\infty$ & $\infty$ & $\infty$ \\
FSACC $^{C}$ (The Americas) & 1 & 1 & 0.9813 & 0.9230 & 0.7454 & 0.3849 & 0.2182 & 0.4082 & $\infty$ & $\infty$ \\
FSACC $^{C}$ (Europe) & 1 & 1 & 1 & 0.9864 & 0.8699 & 0.4932 & 0.2357 & $\infty$ & $\infty$ & $\infty$ \\
FSACC $^{C}$ (Sub-Saharan Africa) & 0.9891 & 0.9668 & 0.8470 & 0.5108 & 0 & $\infty$ & $\infty$ & $\infty$ & $\infty$ & $\infty$ \\
\hline
\end{tabular}

Table 30

Thus, The Americas and Europe have the highest correlation levels in this case.

\section{Sociological views on vulnerability and government responses to human trafficking}

Human slavery is probably present in all nations, but to highly varying degrees. Between-nation trafficking networks involve almost all nations as either sources or destinations, but to highly varying degrees. The United Nations report focuses on factors that make some nations more vulnerable as sources of slaves, and describes variations in national public-policy responses to inter-national trafficking across nations. Both concepts - vulnerability and government response - are complex. Whether a nation is seen as vulnerable or not, and whether that nation's governmental response is seen as extensive or not, are uncertain due to definitional complexity. Assessing the general magnitude of the association between vulnerability and governmental response, measured across nations, is therefore also made uncertain because of the complexity of the concepts.

Many of the conditions that are identified as indications of vulnerabil- 
ity to human trafficking (weak civil and political protections; limited social, health, and economic rights; personal insecurity; and high levels of refugees and conflict), may both produce a need for governmental response and, at the same time, limit the capacity of government to respond. Governmental responses may be of multiple sorts, including survivor support, criminal justice, coordination and accountability, and risk assessment. Overall, across all nations, and by most measures, there is a negative association between vulnerability and response. That is, nations that display greater vulnerability to involvement in international human trafficking also display weaker public policy responses. The correlation, however, is far from perfect, and may vary across regions.

Because of geographical, cultural, and other factors, the strength of the association may differ from region to region. In some regions, certain combinations of factors producing vulnerability may be more common than in other regions; yet mean vulnerabilities may be similar. In some regions patterns of governmental responses may differ from the patterns in other regions; yet the mean responsiveness might be regarded as similar. To assess whether the association between the two concepts is equally strong across regions, methods are needed to deal with definitional uncertainty. We shall assume that other sources of uncertainty sampling and instrumentation are absent.

\section{Fuzzy soft set analysis compared to "conventional" anal- ysis in sociology}

"The Global Slavery Index 2016" [4] data on four parameters of vulnerability and five parameters of government response for 167 nations, are divided into six regions. The five government responsiveness parameters (f) are measured quantitatively and is scaled such that high scores indicate greater responsiveness. The four vulnerability parameters (e) are measured quantitatively as scores on factors from 24 indicators, and is scaled such that high scores indicate greater vulnerability. One seeks to examine the distribution of case's vulnerabilities and responsiveness, as well as the association between these two concepts across the universe of nations (and within regions). Table 31 shows the raw data for the Asia region. We see, for examples, that Australia ranks high on responsiveness, but low on vulnerability; Afghanistan displays high levels of vulnerability and low responsiveness 


\begin{tabular}{|c|c|c|c|c|c|c|c|c|c|}
\hline Name & $f_{1}$ & $f_{2}$ & $f_{3}$ & $f_{4}$ & $f_{5}$ & $e_{1}$ & $e_{2}$ & $e_{3}$ & $e_{4}$ \\
\hline Afghanistan & 0 & 0 & 0 & 0 & 0 & 83 & 47.39 & 53.31 & 84.55 \\
Australia & 64.44 & 81.85 & 56.25 & 69.05 & 25 & 15.14 & 19.85 & 17.45 & 35.49 \\
Bangladesh & 39.44 & 60.37 & 68.75 & 59.52 & 0 & 46.78 & 46.04 & 33.63 & 50.02 \\
Brunei & 7.41 & 35.74 & 12.5 & 30.95 & 0 & 60.78 & 30.99 & 99.99 & 63.93 \\
Cambodia & 25.19 & 33.33 & 37.5 & 64.29 & 0 & 53.68 & 42.96 & 57.4 & 12 \\
China & 35.56 & 23.7 & 31.25 & 52.38 & 0 & 55.12 & 26.9 & 43.84 & 52.78 \\
HSC & 5.93 & 5.19 & 0 & 30.95 & 0 & 42.28 & 17.55 & 21.44 & 35.65 \\
India & 44.07 & 45 & 43.75 & 45.24 & 0 & 37.07 & 36.68 & 43.88 & 87.78 \\
Indonesia & 37.59 & 40.56 & 50 & 54.76 & 0 & 39.15 & 43.35 & 50.38 & 36.01 \\
Japan & 42.59 & 19.44 & 18.75 & 45.24 & 0 & 25.23 & 19.09 & 22.16 & 19.13 \\
Laos & 28.7 & 26.48 & 31.25 & 50 & 0 & 56.64 & 34.01 & 53.98 & 1.19 \\
Malaysia & 36.48 & 51.48 & 31.25 & 35.71 & 0 & 34.9 & 32.43 & 46.39 & 40.33 \\
Mongolia & 27.78 & 31.67 & 31.25 & 47.62 & 0 & 39.22 & 36.74 & 40.87 & 3.54 \\
Myanmar & 41.11 & 8.89 & 50 & 50 & 0 & 57.81 & 50.11 & 50.53 & 66.99 \\
Nepal & 42.78 & 38.15 & 75 & 61.9 & 0 & 42.3 & 43.22 & 34.74 & 41.21 \\
New Zealand & 53.7 & 47.96 & 43.75 & 88.1 & 0 & 13.31 & 22.24 & 16.09 & 21.51 \\
North Korea & 0 & 13.89 & 0 & -7.14 & 0 & 71.2 & 48.27 & 62.88 & 1 \\
Pakistan & 28.52 & 37.04 & 25 & 76.19 & 0 & 58.4 & 41.98 & 52.7 & 96.79 \\
PNG & 6.48 & 23.7 & 25 & 14.29 & 0 & 50.12 & 62.85 & 99.99 & 23.1 \\
Philippines & 46.48 & 62.78 & 50 & 78.57 & 0 & 44.76 & 39.62 & 52.34 & 53.95 \\
Singapore & 36.11 & 22.41 & 0 & 42.86 & 0 & 29.85 & 22.11 & 20.58 & 1 \\
South Korea & 35.93 & 31.85 & 12.5 & 33.33 & 0 & 38.2 & 34.64 & 28.98 & 17.32 \\
Sri Lanka & 25.93 & 38.52 & 37.5 & 83.33 & 0 & 47.01 & 35.12 & 31.82 & 31.08 \\
Taiwan & 50.56 & 23.62 & 43.75 & 42.86 & 0 & 34.91 & 33.38 & 22.34 & 1.76 \\
Thailand & 35.19 & 35.93 & 56.25 & 61.9 & 0 & 49.23 & 28.62 & 48.97 & 63.33 \\
Timor-Leste & 25.93 & 25.93 & 0 & 40.48 & 0 & 38.88 & 48.07 & 68.55 & 1 \\
Vietnam & 45.19 & 34.07 & 62.5 & 66.67 & 0 & 51.19 & 29.94 & 35.22 & 1 \\
\hline
\end{tabular}

Table 31 
678 S. Acharjee, D.J. Sarma, R.A. Hanneman, J.N. Mordeson and D.S. Malik

Table 32 shows Pearson's zero-order product-moment correlations.

\begin{tabular}{|c|c|c|c|c|c|c|c|c|c|}
\hline & $f_{1}$ & $f_{2}$ & $f_{3}$ & $f_{4}$ & $f_{5}$ & $e_{1}$ & $e_{2}$ & $e_{3}$ & $e_{4}$ \\
\hline$f_{1}$ & 1.00 & & & & & & & & \\
$f_{2}$ & 0.68 & 1.00 & & & & & & & \\
$f_{3}$ & 0.68 & 0.63 & 1.00 & & & & & & \\
$f_{4}$ & 0.71 & 0.69 & 0.66 & 1.00 & & & & & \\
$f_{5}$ & 0.39 & 0.50 & 0.21 & 0.18 & 1.00 & & & & \\
$e_{1}$ & -0.72 & -0.57 & -0.25 & -0.47 & -0.39 & 1.00 & & & \\
$e_{2}$ & -0.39 & -0.21 & 0.04 & -0.32 & -0.30 & 0.55 & 1.00 & & \\
$e_{3}$ & -0.62 & -0.21 & -0.24 & -0.41 & -0.26 & 0.59 & 0.65 & 1.00 & \\
$e_{4}$ & -0.05 & 0.16 & 0.16 & 0.08 & 0.00 & 0.32 & 0.13 & 0.19 & 1.00 \\
\hline
\end{tabular}

Table 32

The associations among government responsiveness indicators (f) are positive, substantial, and similar to one another. The associations among the indictors of vulnerability (e) also tend to be positive, but display a great deal of variation. Nations that are high on one aspect of vulnerability are not necessarily high on others. The associations between the set of responsiveness indicators and the set of vulnerability indicators are generally negative, consistent with the notion that many of the same factors that produce vulnerability to human trafficking, may limit governmental responses to it. The associations are far from homogeneous.

Overall, the pattern of associations, as measured by traditional "hard" statistics, suggest that, if we were to divide nations in to "high-risk" versus "low-risk" for trafficking, we would get different answers depending on which parameter we used. The same is true for classifying national government responses. It follows that the strength of the association between the two concepts depends considerably on which parameters are examined.

A conventional approach to summarizing the overall pattern of association between the two sets of indicators is to identify components or factors in each indicator, and to examine the correlation between the factors. For the 27 nations in the Asia region, the $1^{\text {st }}$ canonical correlation is 0.85 , suggesting a very strong association between the underling concepts of vulnerability and governmental response. 
How certain should we be, on the basis of such analysis that there really is a strong negative association between vulnerability and governmental responsiveness to human trafficking across the Asian nations? There are many heroic assumptions built into the conventional "hard" statistical approach.

\section{References}

[1] S. Acharjee and J. N. Mordeson, Soft statistics with respect to utility and application to human trafficking, New Math. Nat. Comput. 13(03), pp. 289-310, (2017).

[2] N. Çăğman, S. Karataş and S. Enginoglu, Soft topology, Comput. Math. Appl., 62, pp. 351-358, (2011).

[3] D. Chen, The parametrization reduction of soft sets and its application, Comput. Math. Appl., 49, pp. 757-763, (2005).

[4] http://www.globalslaveryindex.org/, The Global Slavery index, (2016).

[5] http://www.unodc.org/undoc/en/humantrafficking/ whatishumantrafficking, United Nations Office on Drug and Crime

[6] Y. Leung, A fuzzy set analysis of sociometric structure, Jour. Math. Socio. 7, pp. 159-180, (1980).

[7] H. Li and Y. Shen, Similarity measures of fuzzy soft sets based on different distances, Fifth Int. Symp. Comput. Intel. and Design, pp. 527- 529, (2012).

[8] S. Li, Measuring the fuzziness of human thoughts: an application of fuzzy sets to sociological research, Jour. Math. Socio. 14(1), pp. 67-84, (1989).

[9] P. K. Maji, R. Biswas and A. R. Roy, Fuzzy soft sets, Jour. Fuzzy Math. 9(3), pp. 589-602, (2001).

[10] P. K. Maji, R. Biswas and A. R. Roy, Soft set theory, Comput. Math. Appl. 45, pp. 555-562, (2003). 
680 S. Acharjee, D.J. Sarma, R.A. Hanneman, J.N. Mordeson and D.S. Malik

[11] P. Majumdar and S. K. Samanta, On similarity measures of fuzzy soft sets, Int. Jour. Adv. Soft. Comput. Appl. 3(2), 8pages, (2011).

[12] D. Molodtsov, Soft set theory-first results, Comput. Math. Appl. 37, pp. 19-31,(1999).

[13] J. N. Mordeson, M. Mallenby, S. Mathew and S. Acharjee, Human trafficking: Policy intervention, New Math. Nat. Comput. 13(03), pp. 341-358, (2017).

[14] D. Pei and D. Miao, From soft sets to information systems, Proceedings of the IEEE international conference on granular computing, 2, pp. 617-621, (2005).

[15] Trafficking in Persons: Global Patterns, United Nations Office on Drugs and Crime, Trafficking in Persons Citation Index.

[16] Z. Xiao and Y. Zou, A comparative study of soft sets with fuzzy sets and rough sets, Jour. Intel. Fuzzy Syst. 27, pp. 425-434, (2014).

[17] L. A. Zadeh, Fuzzy sets, Inform. Control. 8(3), pp. 338-353, (1965).

\section{Santanu Acharjee}

Economics and Computational Rationality Group

Department of Mathematics

Debraj Roy College

Golaghat-785621, Assam

India

e-mail : sacharjee326@gmail.com

\section{Diganta Jyoti Sarma}

Department of Mathematics

Central Institute of Technology

BTAD, Kokrajhar-783370, Assam

India

e-mail : dj.sarma@cit.ac.in 


\section{Robert A. Hanneman}

Department of Sociology

University of California

Riverside California-92521

U. S. A.

e-mail : hanneman@ucr.edu

\section{John N. Mordeson}

Center for Mathematics of Uncertainty

Department of Mathematics

Creighton University

Omaha, Nebraska, U. S. A.

e-mail : JohnMordeson@creighton.edu

and

\section{Davender S. Malik}

Center for Mathematics of Uncertainty

Department of Mathematics

Creighton University

Omaha, Nebraska

U. S. A.

e-mail : DavenderMalik@creighton.edu 